TRANSACTIONS OF THE

AMERICAN MATHEMATICAL SOCIETY

Volume 354, Number 8, Pages 3031-3057

S 0002-9947(02)03011-8

Article electronically published on April 3, 2002

\title{
BIRATIONAL AUTOMORPHISMS OF QUARTIC HESSIAN SURFACES
}

\author{
IGOR DOLGACHEV AND JONGHAE KEUM
}

\begin{abstract}
We find generators of the group of birational automorphisms of the Hessian surface of a general cubic surface. Its nonsingular minimal model is a K3 surface with the Picard lattice of rank 16 which embeds naturally in the even unimodular lattice $I I_{1,25}$ of rank 26 and signature $(1,25)$. The generators are related to reflections with respect to some Leech roots. A similar observation was made first in the case of quartic Kummer surfaces in the work of Kondō. We shall explain how our generators are related to the generators of the group of birational automorphisms of a general quartic Kummer surface which is birationally isomorphic to a special Hessian surface.
\end{abstract}

\section{INTRODUCTION}

Let $S: F\left(x_{0}, x_{1}, x_{2}, x_{3}\right)=0$ be a nonsingular cubic surface in $\mathbb{P}^{3}$ over $\mathbb{C}$. Its Hessian surface is a quartic surface defined by the determinant of the matrix of second-order partial derivatives of the polynomial $F$. When $F$ is general enough, the quartic $H$ is irreducible and has 10 nodes. It contains also 10 lines which are the intersection lines of five planes in general linear position. The union of these five planes is classically known as the Sylvester pentahedron of $S$. The equation of $S$ can be written as the sum of cubes of some linear forms defining the five planes. A nonsingular model of $H$ is a K3-surface $\tilde{H}$. Its Picard number $\rho$ satisfies the inequality $\rho \geq 16$. In this paper we give an explicit description of the $\operatorname{group} \operatorname{Bir}(H)$ of birational isomorphisms of $H$ when $S$ is general enough so that $\rho=16$. Although $H$, in general, does not have any non-trivial automorphisms (because $S$ does not), the group $\operatorname{Bir}(H) \cong \operatorname{Aut}(\tilde{H})$ is infinite. We show that it is generated by the automorphisms defined by projections from the nodes of $H$, a birational involution which interchanges the nodes and the lines, and the inversion automorphisms of some elliptic pencils on $\tilde{H}$. This can be compared with the known structure of the group of automorphisms of the Kummer surface associated to the Jacobian abelian surface of a general curve of genus 2 (see [Ke2], [Ko]). The latter surface is birationally isomorphic to the Hessian $H$ of a cubic surface ([Hu1]), but the Picard number of $\tilde{H}$ is equal to 17 instead of 16 . We use the method for computing $\operatorname{Bir}(H)$ employed by Kondō in [Ko]. We show that the Picard lattice $S_{H}$ of the K3-surface $\tilde{H}$ can be primitively embedded into the unimodular lattice $L=\Lambda \perp U$ of signature

Received by the editors June 30, 2001 and, in revised form, August 27, 2001.

2000 Mathematics Subject Classification. Primary 14J28, 14J50; Secondary 11 H56.

Key words and phrases. Quartic Hessian surfaces, automorphisms, K3 surface, Leech lattice. Research of the first author was partially supported by NSF grant DMS 9970460.

Research of the second author was supported by Korea Research Foundation Grant KRF-2000041-D00014. 
$(1,25)$, where $\Lambda$ is the negative definite Leech lattice and $U$ is the hyperbolic plane. The orthogonal complement of $S_{H}$ in $L$ is a primitive lattice of rank 10 which contains a negative definite root lattice of type $A_{5}+A_{1}^{5}$. The fundamental domain $D$ of the reflection group of $L$ cuts out a finite polyhedron $D^{\prime}$ in the fundamental domain $D_{H}$ for the (-2)-reflection group of $S_{H}$ in the connected component of the set $\left\{x \in S_{H} \otimes \mathbb{R}:(x, x)>0\right\}$ containing an ample divisor class. We determine the hyperplanes $h$ which bound $D^{\prime}$ and match them with automorphisms $h \rightarrow \sigma_{h}$ of $\tilde{H}$ such that $\sigma_{h}$ sends one of the half-spaces defined by $h$ to the other half-space defined by $h$ or to one of the two half-spaces corresponding to $\sigma_{h}^{-1}$. This allows one to prove that the automorphisms $\sigma_{h}$ generate a group of symmetries of $D_{H}$, having $D^{\prime}$ as its fundamental domain. By the Torelli Theorem for K3-surfaces this implies that the automorphisms $\sigma_{h}$ together with some symmetries of $D^{\prime}$ generate the group $\operatorname{Aut}(\tilde{H})$. The reason why the beautiful combinatorics of the Leech lattice plays a role in the description of the automorphisms of $H$ is still unclear to us. We hope that the classification of all K3-surfaces whose Picard lattice is isomorphic to the orthogonal complement of a root sublattice of $L$ will shed more light on this question (for such K3 surfaces, also see [KK]).

\section{Some Classical Facts}

Here we summarize without proofs some of the known properties of the quartic Hessian surface $H$ of a cubic surface $F$ in $\mathbb{P}^{3}$. We refer for the proofs to the classical literature (for example, $\mathrm{Ba}]$, $\mathrm{Sa}]$ ).

(1) $H$ is the locus of points $x \in \mathbb{P}^{3}$ such that the polar quadric $P_{x}(F)$ of $F$ is singular.

(2) $H$ is the locus of points $x \in \mathbb{P}^{3}$ such that there exists a polar quadric $P_{y}(F)$ of $F$ such that $x \in \operatorname{Sing}\left(P_{y}(F)\right)$.

(3) If $F$ is nonsingular and general enough, then $H$ has 10 nodes corresponding to polar quadrics of corank 2 and has 10 lines corresponding to their singular lines.

(4) The lines (resp. singular points) of $H$ are the intersection lines of 10 pairs (resp. 10 triples) of hyperplanes $\pi_{i}, i=1, \ldots, 5$, any four of them being linearly independent. The union of the planes $\pi_{i}$ is called the Sylvester pentahedron of the cubic surface.

(5) If $l_{i}=0$ are the equations of the hyperplanes $\pi_{i}$, the equation of $F$ can be written in the Sylvester form $a_{1} l_{1}^{3}+\ldots+a_{5} l_{5}^{3}=0$. The equation of $H$ can be written in the form $\frac{1}{a_{1} l_{1}}+\ldots+\frac{1}{a_{5} l_{5}}=0$.

(6) $H \cap F$ is the parabolic curve of $F$ : the set of points $x \in F$ such that the tangent hyperplane $T_{x}(F)$ intersects $F$ along a cubic curve which has a cuspidal point at $x$.

(7) If $x \in H$ and $y \in \operatorname{Sing}\left(P_{x}(F)\right)$, then the correspondence $x \rightarrow y$ is a birational involution $\tau$ of $H$. On a minimal nonsingular model $\tilde{H}$ it interchanges the exceptional curve blown up from the node $P_{i j k}=\pi_{i} \cap \pi_{j} \cap \pi_{k}$ with the line $L_{l m}=\pi_{l} \cap \pi_{m}$, where $\{i, j, k, l, m\}=\{1,2,3,4,5\}$. The involution $\tau$ of $\tilde{H}$ is fixed-point-free and its quotient $Y=\tilde{H} /(\tau)$ is an Enriques surface. The pair of points in the involution defines a line in $\mathbb{P}^{3}$. The set of such lines forms the Reye congruence of lines isomorphic to $Y$ (see $[\mathrm{Cos}]$ ). 
(8) The pencil of planes passing through a line $L_{i j}$ of $H$ cuts out a pencil of cubic curves on $H$. The plane touching $H$ along the line $L_{i j}$ defines a conic $C_{i j}$ on $H$.

(9) The union of the conics corresponding to the four lines lying in the same plane $\pi_{i}$ is cut out by a quadric.

(10) Projecting from a node $P_{i j k}$, we get a representation of $H$ as the double cover of $\mathbb{P}^{2}$ branched along the union of two cubics. The two cubics intersect at the six vertices of a complete quadrilateral $x y z(x+y+z)=0$, tangent at three vertices $(0,0,1),(0,1,0),(1,0,0)$ with the tangent directions such that there exists a conic intersecting each cubic only at these three points.

\section{The Reye COngruence}

The Hessian surface $H$ is a special case of a quartic symmetroid, that is, a quartic surface given by vanishing of the determinant of a symmetric matrix with linear homogeneous polynomials as its entries. We refer to [Cos] for general properties of quartic symmetroids and the associated Reye congruences of lines.

Let us look closer at the Enriques surface $Y=\tilde{H} /(\tau)$. We have a natural indexing of faces $\pi_{i}$ of the Sylvester pentahedron by the set $\{1,2,3,4,5\}$, edges by subsets $\alpha=\{i, j\} \subset\{1,2,3,4,5\}$, and the vertices by subsets $\beta=\{i, j, k\} \subset\{1,2,3,4,5\}$. A vertex $P_{i j k}$ belongs to an edge $L_{m n}$ if and only if $\{i, j, k\} \supset\{m, n\}$. Let $N_{\alpha}$ be the smooth rational curve on $\tilde{H}$ corresponding to $P_{\alpha}$ and similarly let $T_{\beta}$ be the smooth rational curve corresponding to $L_{\beta}$. The Enriques involution $\tau$ interchanges the curves $N_{\alpha}$ and $T_{\beta}$, where $\alpha$ and $\beta$ are complementary sets. Let $U_{\beta}$ be the image on $Y$ of the pair $\left(N_{\alpha}, T_{\beta}\right)$. We have

$$
U_{a b} \cdot U_{c d}= \begin{cases}1, & \text { if }\{c, d\} \cap\{a, b\}=\emptyset ; \\ 0, & \text { otherwise. }\end{cases}
$$

The dual intersection graph is the famous Petersen tri-valent graph with group of symmetries isomorphic to the permutation group $S_{5}$.

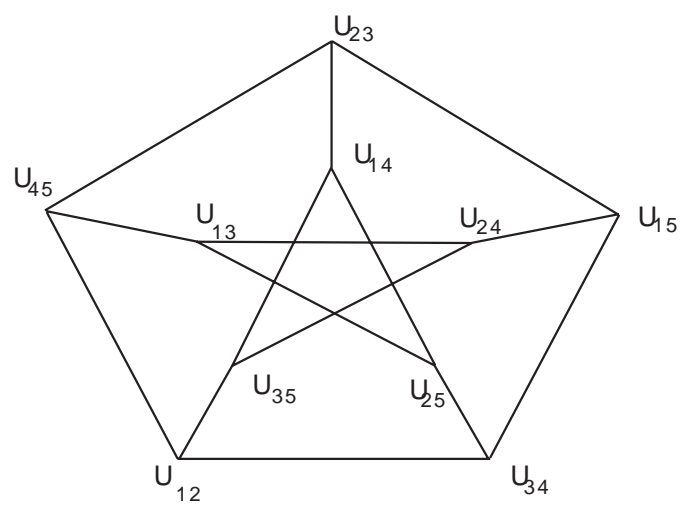

FiguRe 1.

The sum $\Delta$ of the nodal curves $U_{a b}$ satisfies $\Delta^{2}=10$. It defines an embedding of $Y$ into the Grassmann variety $G(2,4)$ which exhibits $Y$ as the Reye congruence of lines for the web of polar quadrics to $F$. 
Let $D_{12}=U_{12}+U_{34}+U_{35}+U_{45}$ and $F_{12}=\Delta-D_{12}$. Then $\left|F_{12}\right|$ is an elliptic pencil on $Y$ with degenerate fibre $F_{12}$ of Kodaira type $I_{6}$ and one fibre of type $I_{2}$ formed by $U_{12}$ and the image of the conic $C_{12}$. Similarly we define other elliptic pencils $\left|F_{a b}\right|$. There are ten such elliptic pencils on $Y$. We have $F_{a b} \cdot F_{c d}=4$ if $\{c, d\} \neq\{a, b\}$. Note that

$$
\sum_{a b} F_{a b}=6 \Delta, \quad \sum_{a b} E_{a b}=3 \Delta,
$$

where $2 E_{a b}$ is a multiple fibre of $\left|F_{a b}\right|$. If $U_{a b}$ and $U_{c d}$ intersect, the sum $D_{a b}+D_{c d}$ is a degenerate fibre of type $I_{2}^{*}=\tilde{D}_{5}$ of the elliptic pencil $\left|2 \Delta-F_{a b}-F_{c d}\right|=$ $\left|D_{a b}+D_{c d}\right|$. The linear system $\left|F_{a b}+F_{c d}\right|$ defines a finite map of degree 2 from $Y$ to a Del Pezzo surface $\mathcal{D}_{4}$ of degree 4 . It blows down two Dynkin curves of type $A_{2}$. The ramification curve is of genus 3 .

The pull-backs of the elliptic pencils on $Y$ to $\tilde{H}$ give elliptic pencils of two types: Type I with degenerate fibres $2 I_{6}+2 I_{2}$ and Type II with degenerate fibres $2 I_{2}^{*}$. A pencil of Type I is cut out by planes through a line $L_{i j}$ or, in the double plane construction, by the pencil of cubic curves spanned by the components of the branch locus.

\section{The Picard lattice}

Let us consider the double plane construction of $H$ and the corresponding elliptic pencil. The surface $\tilde{H}$ admits a double cover $\pi: \tilde{H} \rightarrow V$ to a rational elliptic surface $V$ obtained by blowing up the base points of the pencil of cubic curves spanned by the components of the branch locus. It is branched along two smooth elliptic fibres. The elliptic fibration $f: V \rightarrow \mathbb{P}^{1}$ has one reducible fibre of type $I_{6}$ and one of type $I_{2}$. Let $E_{i}, i=0, \ldots, 5$, be the irreducible components of the fibre of type $I_{6}$, and let $E_{6}$ be one of the irreducible components of the fibre of type $I_{2}$.

3.1. Lemma. The Picard group $\operatorname{Pic}(\tilde{H})$ is generated by $\pi^{*}(\operatorname{Pic}(V))$ and the divisor classes of the curves $E_{i}, i=0, \ldots, 6$.

Proof. Consider the pull-back $f^{\prime}: \tilde{H} \rightarrow \mathbb{P}^{1}$ of the elliptic fibration $f$ with the base obtained by the double cover of $\mathbb{P}^{1}$ ramified at two points. It has two reducible fibres of type $I_{6}$ and two of type $I_{2}$. The Mordell-Weil group MW $(f)$ of $f$ is of rank 2 and is isomorphic to the Mordell-Weil group MW $\left(f^{\prime}\right)$ of $f^{\prime}$. This can be seen as follows. Since the Picard number of $\tilde{H}$ is equal to 16 , the rank of $\operatorname{MW}\left(f^{\prime}\right)$ is equal to $16-10-2-2=2$. Let us choose the zero section of $f^{\prime}$ to be the pre-image of the zero section of $f$. Then the covering involution $\alpha$ of the double cover $\pi: \tilde{H} \rightarrow V$ defines an automorphism of the group $\mathrm{MW}\left(f^{\prime}\right)$ which is identical on $\pi^{*}(\mathrm{MW}(f))$. Suppose we have a section $s \in \mathrm{MW}\left(f^{\prime}\right) \backslash \pi^{*}(\mathrm{MW}(f))$. Since MW $\left(f^{\prime}\right) / \pi^{*}(\mathrm{MW}(f))$ is a group of finite order, there exists an integer $n$ such that $n s \in \pi^{*}(\mathrm{MW}(f))$ and hence $n \alpha(s)=\alpha(n s)=n s$. This implies that the section $\alpha(s)-s$ is of finite order and intersects the zero section at the points of the ramification divisor of $\pi$. However, it is well known that any two different torsion sections of an elliptic fibration are disjoint.

It is known that the Picard group of an elliptic surface is generated by sections and components of fibres. By the previous argument, any section on $\tilde{H}$ is a preimage of a section on $V$. The sum $\sum_{i=0}^{5} E_{i}$ is a fibre of $f^{\prime}$. Since $E_{i}+\alpha\left(E_{i}\right)$ is the pre-image of a component of a fibre of $f$, we obtain that any divisor class on $\tilde{H}$ can 
be written as a sum $D_{1}+D_{2}$, where $D_{1}$ is the pre-image of a divisor class on $V$ and $D_{2}$ is a linear combination of the divisor classes of the curves $E_{i}, i=0, \ldots, 6$. This proves the lemma.

3.2. Corollary. The Picard group $\operatorname{Pic}(\tilde{H})$ is generated over integers by the twenty smooth rational curves $N_{\alpha}$ and $T_{\beta}$.

Proof. We have seen already that $\operatorname{Pic}(\tilde{H})$ is generated by $\pi^{*}(\operatorname{Pic}(V))$ and the curves $E_{i}, i=0, \ldots, 6$. The latter curves belong to the set $N T=\left\{N_{\alpha}, T_{\beta}\right\}$. The group $\operatorname{Pic}(V)$ is generated by the exceptional curves blown up from the vertices of the quadrilateral and the proper transforms of its sides. The pre-images of all these curves in $\tilde{H}$ belong to the set $N T$.

Let $T_{\tilde{H}}$ be the lattice of transcendental cycles on $\tilde{H}$. By definition, it is equal to the orthogonal complement of $\operatorname{Pic}(\tilde{H})$ in the cohomology lattice $L=H^{2}(\tilde{H}, \mathbb{Z})$. Since the latter is a unimodular lattice, the lattices $\operatorname{Pic}(\tilde{H})$ and $T_{\tilde{H}}$ have isomorphic discriminant groups, and the quadratic forms on the discriminant groups differ only by the sign. We are not able to give a formula for $\operatorname{Pic}(\tilde{H})$ in terms of an orthogonal sum of some standard lattices. However, it is possible to give such a formula for the lattice $T_{\tilde{H}}$.

Let $A_{n}$ denote the negative definite root lattice of type $A_{n}$ (it is defined by the matrix equal to the Cartan matrix of the root system of type $A_{n}$ taken with the negative sign), let $U$ be the standard hyperbolic plane, and, for any lattice $M$, let $M(m)$ denote the lattice $M$ with quadratic form multiplied by $m$.

\subsection{Theorem.}

$$
T_{\tilde{H}} \cong U \perp U(2) \perp A_{2}(2) .
$$

Proof. Consider the sublattice $S$ of $\operatorname{Pic}(\tilde{H})$ spanned by $\pi^{*}(\operatorname{Pic}(V))$ and the divisor classes $E_{i}^{*}=\left[E_{i}-\alpha\left(E_{i}\right)\right], i=0, \ldots, 6$. We have $\sum_{i=0}^{5} E_{i}^{*}=0$. This gives

$$
S \cong \pi^{*}(\operatorname{Pic}(V)) \perp \sum_{i=1}^{6} \mathbb{Z}\left[E_{i}^{*}\right] \cong A_{1}(-1) \perp A_{1}^{\perp 9} \perp A_{5}(2) \perp A_{1}(2) .
$$

Note that

$$
2\left[E_{i}\right]=\left(\left[E_{i}+\alpha\left(E_{i}\right)\right]\right)+\left(\left[E_{i}-\alpha\left(E_{i}\right)\right]\right) \in S .
$$

Obviously $\operatorname{Pic}(\tilde{H}) / S$ is generated by the classes $\left[E_{i}\right]$ of the divisors $E_{i}, i=0, \ldots, 6$, which are elements of order 2 . Also these classes are linearly independent. In fact, assume that for some subset $I \subset\{0, \ldots, 6\}$ we have $e_{I}=\sum_{i \in I}\left[E_{i}\right] \in S$. Write $e_{I}$ as a $\operatorname{sum} \pi^{*}(x)+y$, where $x \in \operatorname{Pic}(V), y \in \sum_{i=0}^{6} \mathbb{Z}\left[E_{i}^{*}\right]$. Then

$$
e_{I}+\alpha\left(e_{I}\right)=\sum_{i \in I}\left[E_{i}+\alpha\left(E_{i}\right)\right]=\pi^{*}(2 x) .
$$

However, $E_{i}+\alpha\left(E_{i}\right)=\pi^{*}\left(E_{i}^{\prime}\right)$, where $E_{i}^{\prime}$ is a component of a fibre of the elliptic fibration $f$. It is easy to see that the divisor class of the sum $\sum_{i \in I} E_{i}^{\prime}$ is not divisible by 2 in $\operatorname{Pic}(V)$. Thus we have

$$
\operatorname{Pic}(\tilde{H}) / S \cong(\mathbb{Z} / 2)^{7} .
$$

This shows that the discriminant of the lattice $\operatorname{Pic}(\tilde{H})$ is equal to $2^{18} \cdot 3 / 2^{14}=2^{4} \cdot 3$. Moreover, let us show that the discriminant quadratic form $q_{D}$ of the discriminant 
group $D$ of $\operatorname{Pic}(\tilde{H})$ coincides with minus the discriminant quadratic form of the lattice $U \perp U(2) \perp A_{2}(2)$. We have

$$
S \subset \operatorname{Pic}(\tilde{H}) \subset \operatorname{Pic}(\tilde{H})^{*} \subset S^{*} .
$$

This shows that the group $A=\operatorname{Pic}(\tilde{H}) / S$ is a subgroup of the discriminant group $D(S)$ of $S$ which is isotropic with respect to the discriminant quadratic form. The discriminant group $D$ is equal to $A^{\perp} / A$, where the orthogonal complement means the orthogonal complement with respect to the quadratic form of $D(S)$ (see [Ni]).

Let $s_{0}, E_{1}^{\prime}, s_{1}, s_{2}, E_{3}^{\prime}, s_{3}, s_{4}, E_{5}^{\prime}, s_{5}$ be the 9 curves on $V$ coming from blowing up of $\mathbb{P}^{2}$. Here, $E_{1}^{\prime}, E_{3}^{\prime}, E_{5}^{\prime}$ are $(-2)$-curves contained in the reducible fibre of $f$ of type $I_{6}$, and $s_{0}, s_{1}, \ldots, s_{5}$ are $(-1)$-curves which are sections of $f$. Then $\operatorname{Pic}(V)$ is generated by these nine curves and the full transform $l_{0}$ of a line on $\mathbb{P}^{2}$. The 10 divisor classes

$$
\begin{gathered}
e_{0}=\pi^{*}\left(l_{0}\right), e_{1}=\pi^{*}\left(E_{1}^{\prime}+s_{1}\right), e_{2}=\pi^{*}\left(s_{1}\right), e_{3}=\pi^{*}\left(E_{3}^{\prime}+s_{3}\right), e_{4}=\pi^{*}\left(s_{3}\right), \\
e_{5}=\pi^{*}\left(E_{5}^{\prime}+s_{5}\right), e_{6}=\pi^{*}\left(s_{5}\right), e_{7}=\pi^{*}\left(s_{0}\right), e_{8}=\pi^{*}\left(s_{2}\right), e_{9}=\pi^{*}\left(s_{4}\right),
\end{gathered}
$$

form a standard orthogonal basis of $\pi^{*}(\operatorname{Pic}(V)) \cong A_{1}(-1) \perp A_{1}^{\perp 9}$. The lattice $S$ is generated by these 10 classes and 6 divisor classes $E_{i}^{*}, i=1, \ldots, 6$. The discriminant group $D(S)$ is generated by the cosets of

$$
e_{0} / 2, \ldots, e_{9} / 2, E_{2}^{*} / 2, \ldots, E_{5}^{*} / 2,\left(E_{1}^{*}+2 E_{2}^{*}+3 E_{3}^{*}+4 E_{4}^{*}+5 E_{5}^{*}\right) / 12, E_{6}^{*} / 4 .
$$

We can easily express the generators $\left[E_{i}\right], i=0, \ldots, 6$, of the group $A=\operatorname{Pic}(\tilde{H}) / S$ in terms of these generators;

$$
\begin{gathered}
{\left[E_{1}\right]=\left[E_{1}+\alpha\left(E_{1}\right)\right] / 2+\left[E_{1}-\alpha\left(E_{1}\right)\right] / 2=\pi^{*}\left(E_{1}^{\prime}\right) / 2+E_{1}^{*} / 2} \\
=\left(e_{1}-e_{2}\right) / 2+E_{1}^{*} / 2 .
\end{gathered}
$$

Similarly,

$$
\begin{gathered}
{\left[E_{2}\right]=\left(e_{0}-e_{1}-e_{3}-e_{8}+E_{2}^{*}\right) / 2, \quad\left[E_{3}\right]=\left(e_{3}-e_{4}+E_{3}^{*}\right) / 2,} \\
{\left[E_{4}\right]=\left(e_{0}-e_{3}-e_{5}-e_{9}+E_{4}^{*}\right) / 2, \quad\left[E_{5}\right]=\left(e_{5}-e_{6}+E_{5}^{*}\right) / 2,} \\
{\left[E_{6}\right]=\left(e_{0}-e_{7}-e_{8}-e_{9}+E_{6}^{*}\right) / 2, \quad \sum_{i=0}^{5}\left[E_{i}\right]=\left(3 e_{0}-\sum_{i=1}^{9} e_{i}\right) / 2 .}
\end{gathered}
$$

Now it is straightforward to compute the group $A^{\perp} / A$ and its quadratic form. Indeed, it is generated by four elements

$$
\begin{gathered}
x=\left(E_{1}^{*}+2 E_{2}^{*}+3 E_{3}^{*}+4 E_{4}^{*}+5 E_{5}^{*}\right) / 6, \quad y=E_{6}^{*} / 2, \\
u=\left(e_{7}+e_{8}+E_{1}^{*}\right) / 2, \quad v=\left(e_{1}+e_{3}+e_{5}+e_{6}+E_{2}^{*}\right) / 2,
\end{gathered}
$$

and its quadratic form is isomorphic to

$$
\langle x+y+v, y+u\rangle \oplus\langle u, y+v\rangle \cong-q_{A_{2}(2) \perp U(2)} .
$$

Applying Nikulin's results [Ni], we obtain that there exists a unique (up to isomorphism) lattice of signature $(2,4)$ with the discriminant quadratic form isomorphic to minus the discriminant quadratic form of $\operatorname{Pic}(\tilde{H})$. Hence, the transcendental lattice of $\tilde{H}$ is isomorphic to $U \perp U(2) \perp A_{2}(2)$. This proves the theorem. 
3.4. Remark. By a different method (using a computer) the previous result was independently obtained by B. van Geemen $\mathrm{vGe}$.

We set

$$
\mathcal{N}=\sum_{\alpha} N_{\alpha}, \quad \mathcal{T}=\sum_{\beta} T_{\beta}
$$

Let $\eta_{H}$ be the pre-image on $\tilde{H}$ of the class of a hyperplane section of $H$ and let $\eta_{S}$ be its image under the Enriques involuton $\tau$. It is known (see, for example, Cos, Proposition 2.4.1) that

$$
2 \eta_{H}=3 \eta_{S}-\mathcal{T}, \quad 2 \eta_{S}=3 \eta_{H}-\mathcal{N} .
$$

In particular, the pre-image $\tilde{\Delta}$ of the class $\Delta$ on the Enriques surface can be expressed as

$$
\tilde{\Delta}=\mathcal{T}+\mathcal{N}=\eta_{H}+\eta_{S}
$$

Pick up a face of the Sylvester pentahedron, say $\pi_{5}$. It is immediately checked that $2\left(\eta_{S}-\eta_{H}\right)$ intersects each $N_{\alpha}$ and $T_{\beta}$ with the same multiplicity as the divisor $\left(T_{15}+T_{25}+T_{35}+T_{45}\right)-\left(N_{123}+N_{124}+N_{134}+N_{234}\right)$. This gives a linear relation:

$$
2\left(\eta_{S}-\eta_{H}\right)=\left(T_{15}+T_{25}+T_{35}+T_{45}\right)-\left(N_{123}+N_{124}+N_{134}+N_{234}\right) .
$$

Denote the first bracket by $\mathcal{T}_{5}$ and the second bracket by $\mathcal{N}_{5}$. Similarly introduce $\mathcal{T}_{i}, \mathcal{N}_{i}, i=1, \ldots, 4$, for any other face. We obtain

$$
2\left(\eta_{S}-\eta_{H}\right)=\mathcal{T}_{i}-\mathcal{N}_{i}, \quad i=1, \ldots, 5 .
$$

One can show that the linear system $\left|\eta_{H}+\frac{1}{2}\left(\mathcal{T}_{i}-\mathcal{N}_{i}\right)\right|=\left|\eta_{S}\right|$ defines a standard cubic Cremona involution $\tau_{i}$ with fundamental points at the vertices of the tetrahedron formed by the faces $\pi_{j}, j \neq i$. All these involutions restrict to the Enriques involution on $\tilde{H}$ (cf. [Hu2, p.335).

We shall denote by $C_{i j}$ the pre-image on $\tilde{H}$ of the conic cut out by the plane which is tangent to $H$ along the edge $L_{i j}$. Its divisor class equals

$$
C_{i j}=\eta_{H}-2 T_{i j}-N_{i j k}-N_{i j l}-N_{i j m}
$$

We shall also denote by $R_{k l m}$ the pre-image on $\tilde{H}$ of the cubic cut out by the plane through the edge $L_{i j}$ and the opposite vertex $P_{k l m}$. Its divisor class is equal to

$$
R_{k l m}=\eta_{H}-T_{i j}-N_{i j k}-N_{i j l}-N_{i j m}-N_{k l m} .
$$

One can show $([\underline{\mathrm{Hu}}]$ ) that any rational nonsingular curve of degree $\leq 3$ is one of the described above (on a general $H$ ).

\section{Kummer surfaces and Hessians}

Let $C$ be a genus 2 curve and $\left(p_{1}, \ldots, p_{6}\right)$ its ordered set of Weierstrass points. Then the divisor classes $\mu_{0}=0, \mu_{i j}=\left[p_{i}+p_{j}-2 p_{6}\right], 1 \leq i<j \leq 6$, are the sixteen 2 -torsion points on its Jacobian variety $J$. In the usual way we identify the group of 2 -torsion points $J_{2}$ with the set of 2 -element subsets $\alpha$ of the set $S=\{1,2,3,4,5,6\}$; the zero point corresponds to the empty subset. We shall identify a 2 -element subset $\alpha$ with its complementary subset $S \backslash \alpha$. Then the addition of points corresponds to the symmetric sum $\alpha+\beta$ of subsets. One defines the symplectic bilinear form with values in $\mathbb{F}_{2}$ on $J_{2}$ by

$$
\left(\mu_{\alpha}, \mu_{\beta}\right)=|\alpha \cap \beta| \text { modulo } 2 \text {. }
$$


Let us write elements of the vector space $V=\mathbb{F}_{2}^{4}$ as $2 \times 2$ matrices $\left[\begin{array}{l}\epsilon \\ \eta\end{array}\right]$ with rows in $\mathbb{F}_{2}^{2}$. We define the quadratic form $q_{0}: V \rightarrow \mathbb{F}_{2}$ by $q_{0}\left(\left[\begin{array}{l}\epsilon \\ \eta\end{array}\right]\right)=\epsilon \cdot \eta$, where the dot means the standard dot-product in $\mathbb{F}_{2}^{2}$. The associated symmetric bilinear form is

$$
\left(\left[\begin{array}{l}
\epsilon \\
\eta
\end{array}\right],\left[\begin{array}{l}
\epsilon^{\prime} \\
\eta^{\prime}
\end{array}\right]\right)=\epsilon \cdot \eta^{\prime}+\eta \cdot \epsilon^{\prime}
$$

It is a non-degenerate symplectic form. We define an isomorphism of symplectic spaces $\psi: J_{2} \rightarrow V$ by

$$
\mu_{12} \rightarrow\left[\begin{array}{ll}
1 & 0 \\
0 & 0
\end{array}\right], \quad \mu_{34} \rightarrow\left[\begin{array}{ll}
0 & 1 \\
0 & 0
\end{array}\right], \quad \mu_{16} \rightarrow\left[\begin{array}{ll}
0 & 0 \\
1 & 0
\end{array}\right], \quad \mu_{45} \rightarrow\left[\begin{array}{ll}
0 & 0 \\
0 & 1
\end{array}\right]
$$

Let us identify the span $U_{1}$ of $\mu_{12}, \mu_{16}$ with the set [4] $=\{1,2,3,4\}$ by assigning 0 to $1, \mu_{16}$ to $2, \mu_{12}$ to 3 and $\mu_{12}+\mu_{16}=\mu_{26}$ to 4 . Similarly we identify the span $U_{2}$ of $\mu_{34}, \mu_{45}$ with [4] by assigning 0 to $1, \mu_{45}$ to $2, \mu_{34}$ to 3 and $\mu_{34}+\mu_{45}=\mu_{35}$ to 4 . Each 2-torsion point can be written uniquely as the sum $a+b, a \in U_{1}, b \in U_{2}$, and hence can be identified with the pair $(a, b) \in[4] \times[4]$, or, equivalently, with a dot in the following $4 \times 4$ table:

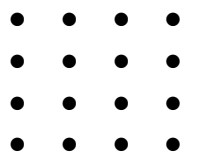

Let $C \rightarrow J, x \rightarrow\left[x-p_{6}\right]$, be the Abel-Jacobi map corresponding to the point $p_{6}$. The image of $C$ is denoted by $\Theta_{0}$. Let $\Theta_{i j}=\Theta_{0}+\mu_{i j}$ be its translate by a 2-torsion point $\mu_{i j}$. Each $\Theta_{i j}$ contains exactly six 2-torsion points, namely the points $\mu_{i j}, \mu_{k 6}+\mu_{i j}, k=1, \ldots, 5$. In other words,

$$
\mu_{\alpha} \in \Theta_{\beta} \Leftrightarrow \beta+\alpha \in\{\emptyset,\{16\},\{26\},\{36\},\{46\},\{56\}\} .
$$

One also employs different indexing of theta divisors $\Theta_{\alpha}$. For each $\alpha$ there exists a partition of $[6]=\{1,2,3,4,5,6\}$ into two disjoint subsets $S \cup S^{\prime}$ with odd numbers of elements. For $\alpha \neq k 6$, it is uniquely determined by the property $\mu_{\beta} \in \Theta_{\alpha}$ if and only if $\beta \subset S$ or $\beta \subset S^{\prime}$. For $\beta=k 6$, we simply take $S=\{k\}$. We use either $S$ or $S^{\prime}$ for the index. In this correspondence, $\Theta_{\beta}$ corresponds to $\Theta_{\beta+\{6\}}$. For example, $\Theta_{12}$ corresponds to $\Theta_{345}$ or $\Theta_{126}$, and $\Theta_{16}$ to $\Theta_{1}$ or $\Theta_{23456}$. Yet there is another classical notation for a theta divisor. Each theta divisor is equal to the set of zeroes of a theta function $\theta\left[\begin{array}{c}{ }^{\prime} \\ \epsilon^{\prime}\end{array}\right](z, \tau)$ with theta characteristic $\left[\begin{array}{c}{ }^{\epsilon} \\ \epsilon^{\prime}\end{array}\right]$. The theta characteristic corresponding to $\Theta_{S}$, where $\# S$ is odd, is equal to $\psi(S+\{135\})$. For example, the theta characteristic corresponding to $\Theta_{12}=\Theta_{345}$ is $\psi(14)=\left[\begin{array}{ll}1 & 1 \\ 1 & 1\end{array}\right]$, where $\epsilon$ and $\epsilon^{\prime}$ are the first and the second rows of the matrix.

One assigns to $\Theta_{\alpha}$ a dot $(a b) \in[4] \times[4]$ in the $4 \times 4$ table as above in such a way that $(a b)$ in the right-side table contains a 2-torsion point corresponding to the

\begin{tabular}{|c|c|c|c|c|c|c|}
\hline$\mu_{0}$ & $\mu_{45}$ & $\mu_{34}$ & $\mu_{35}$ & $\Theta_{12}$ & $\Theta_{36}$ & $\Theta_{56}$ \\
\hline$u_{16}$ & $\mu_{23}$ & $\mu_{25}$ & $\mu_{24}$ & $\Theta_{26}$ & $\Theta_{13}$ & $\Theta_{15}$ \\
\hline 12 & $\mu_{36}$ & $\mu_{56}$ & $\mu_{46}$ & $\Theta_{0}$ & $\Theta_{45}$ & $\Theta_{34}$ \\
\hline & $\mu_{13}$ & $\mu_{15}$ & $\mu_{14}$ & $\Theta_{16}$ & $\Theta_{23}$ & $\Theta_{25}$ \\
\hline
\end{tabular}
entry $(c d)$ in the left-side table if and only if $a=c$ or $b=d$ but $(a b) \neq(c d)$.

Explicitly, we have two tables

The following construction of Hutchinson [Hu1] describes the translation of the left-hand-side table to the table of the corresponding values of the map $\psi$, and, at 
the same time, translates the right-hand-side table to the table of the corresponding theta characteristics. Denote the four columns

$$
\begin{array}{llll}
1 & 1 & 0 & 0 \\
1 & 0 & 1 & 0
\end{array}
$$

by the numbers $1,2,3,4$, respectively. Then the theta characteristic of the $(a b)$ entry in the right-hand-side table is equal to the characteristic formed by the columns $(a b)$. Now, if we reverse the order of the 4 vectors and do the same, we obtain the values of $\psi$ at the entries of the left-hand-side table.

An affine plane in $J_{2}$ is called an odd (or Göpel) tetrad if it is a translation of a totally isotropic linear subspace of dimension 2. Otherwise it is called an even (or Rosenhain) tetrad. There are 60 odd and 80 even tetrads, each set forming an orbit with respect to the group generated by symplectic automorphisms of $J_{2}$ and translations. For example, the rows and columns of the left-hand-side table above correspond to even tetrads but the diagonal corresponds to an odd tetrad.

A Weber hexad is defined as the symmetric sum of an even and an odd tetrad which have one point in common. For example, the dots marked with asterisk represent a Weber hexad:

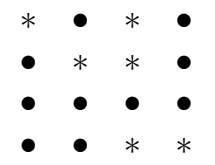

A Weber hexad has the following property. Each theta-divisor contains either 3 points from a Weber hexad or just one. The number of theta divisors which contain three points is equal to ten. One can choose 5 subsets of four elements from this set of ten theta divisors such that each point from the Weber hexad is contained in two theta divisors from this set. For the Weber hexad chosen above, the five sets are the following:

$$
\begin{gathered}
\mathcal{A}_{1}=\left(\Theta_{56}, \Theta_{46}, \Theta_{15}, \Theta_{14}\right), \quad \mathcal{A}_{2}=\left(\Theta_{14}, \Theta_{36}, \Theta_{16}, \Theta_{34}\right), \\
\mathcal{A}_{3}=\left(\Theta_{23}, \Theta_{25}, \Theta_{56}, \Theta_{36}\right), \quad \mathcal{A}_{4}=\left(\Theta_{23}, \Theta_{26}, \Theta_{34}, \Theta_{46}\right), \\
\mathcal{A}_{5}=\left(\Theta_{26}, \Theta_{16}, \Theta_{25}, \Theta_{15}\right) .
\end{gathered}
$$

By inspection, one sees that the theta characteristics of theta functions from the same group of four add up to zero. This means that the sum of the divisors belongs to the linear system $|4 \Theta|$, where $\Theta=\Theta_{24}$ has the zero theta characteristic. Thus we have five divisors $D_{1}, \ldots, D_{5} \in|4 \Theta|$, each passing through every point of the Weber hexad with multiplicity 2 . Let $\theta_{i}, i=0, \ldots, 4$, be the corresponding theta functions of order 4 with zero theta characteristic. They define a rational map $\Phi: J \rightarrow \mathbb{P}^{4}$.

4.1. Theorem. The image $H$ of $\Phi$ is contained in a linear hyperplane $x_{4}=a x_{0}+$ $b x_{1}+c x_{2}+d x_{3}$, where $a, b, c, d \neq 0$, and satisfies the quartic equation

$$
a x_{1} x_{2} x_{3} x_{4}+b x_{0} x_{2} x_{3} x_{4}+c x_{0} x_{1} x_{3} x_{4}+d x_{0} x_{1} x_{2} x_{4}+x_{0} x_{1} x_{2} x_{3}=0 .
$$

In particular, $H$ is isomorphic to the Hessian of a nonsingular cubic surface.

We refer for the proof to [Hu1] or vGe. One can also find there the expression of the coefficients $a, b, c, d$ in terms of theta constants. 
Since $\Theta$ is a symmetric theta divisor, the rational map $\Phi$ factors through the map $J \rightarrow \operatorname{Kum}(J) \subset \mathbb{P}^{3}$ given by the linear system $|2 \Theta|$. The image of the Weber hexad in the Kummer surface is a set of 6 nodes, a Weber set of nodes. The linear system $|4 \Theta|$ is equal to the inverse transform of a linear system $\left|L_{h}\right|$ of quadrics through the Weber set of nodes $h$. Thus the Hessian surface $H=\operatorname{Im}(\Phi)$ is the birational image of $\operatorname{Kum}(J)$ under the map $\bar{\Phi}$ given by the linear system $\left|L_{h}\right|$. An explicit equation of the locus of the cubic surfaces whose Hessian is birationally isomorphic to a Kummer surface inside of the moduli space of cubic surfaces has been found in $[\mathrm{Ro}, \mathrm{vGe}$.

Let us choose the Weber hexad as above:

$$
h=\left(\mu_{0}, \mu_{23}, \mu_{34}, \mu_{25}, \mu_{15}, \mu_{14}\right) .
$$

Let

$$
\left(\Theta_{56}, \Theta_{46}, \Theta_{15}, \Theta_{14}, \Theta_{36}, \Theta_{16}, \Theta_{34}, \Theta_{23}, \Theta_{25}, \Theta_{26}\right)
$$

be the corresponding set of ten theta divisors which contain exactly three of the points from $h$ and hence three points from the set $J_{2} \backslash h$. The images of each node from $h$ under the map $\Phi$ form a conic in $H$. The plane section of $H$ along such a conic is equal to the union of two conics. These conics do not appear on a general Hessian.

The images of the remaining nodes which are the images of ten 2-torsion points $\mu_{\alpha}$ are ten nodes of the Hessian which we denote by $N_{\alpha}$. The image of a theta divisor $\Theta_{\beta}$ from (4.4) is a line on $H$ which contains three nodes $N_{\alpha}$ such that $\mu_{\alpha} \in \Theta_{\beta}$. We denote this line by $T_{\beta}$. Note that each $\Theta_{\beta}$ belongs to exactly two of the five subsets from (4.2), say $\mathcal{A}_{i}, \mathcal{A}_{j}$. If we reindex $T_{\beta}$ by $T_{i j}$ and $N_{\alpha}=N_{i j k}$, where $N_{\alpha} \in T_{i j}, T_{j k}, T_{i k}$, then we get the notation used for the nodes and the lines on a general Hessian. The image of a theta divisor not belonging to the group of ten is a rational curve of degree 3 on $H$ which passes through 5 nodes. These curves do not appear on a general Hessian.

4.2. Remark. A Weber set $h$ of nodes of a Jacobian Kummer surface $K$ is a set $\mathcal{P}$ of 6 points in $\mathbb{P}^{3}$ in general linear position. Recall that each such set defines the Weddle quartic surface $W(\mathcal{P})$ which is the locus of singular points of quadrics passing through the set $\mathcal{P}$. The image of $W(\mathcal{P})$ by the linear system of quadrics through $\mathcal{P}$ is a Kummer surface $K(\mathcal{P})$ birationally isomorphic to $W(\mathcal{P})$. The image of $K$ is a Hessian quartic $H$. The quartic surfaces $K(\mathcal{P})$ and $H$ touch each other along a curve of degree 8 not passing through their nodes. There are 192 Weber hexads. The affine symplectic group $2^{4} \rtimes \mathrm{Sp}\left(4, \mathbb{F}_{2}\right)$ of order $2^{4} \cdot 6$ ! acts transitively on the set of Weber hexads. The isotropy group of a Weber hexad is isomorphic to the alternating group $A_{5}$. Let $\mathcal{M}_{\mathrm{Kum}}^{w}$ be the moduli space of Jacobian Kummer surfaces together with a choice of a Weber hexad of nodes. It is a cover of degree 12 of the moduli space $\mathcal{M}_{2}$ of genus 2 curves isomorphic to the moduli space $\mathcal{M}_{\text {Kum }}$ of Jacobian Kummer surfaces. The above construction defines a map $\mathcal{M}_{\mathrm{Kum}}^{w} \rightarrow$ $\mathcal{M}_{\text {Kum. }}$. What is the degree of this map?

4.3. Remark. Since the K3-surface birationally isomorphic to the Hessian quartic of a nonsingular cubic surface admits an Enriques involution, any K3-surface birationally isomorphic to a Jacobian Kummer surface admits an Enriques involution. This fact, of course, is known, and it is also true for not necessarily Jacobian 
Kummer surfaces (see [Ke1]). But here we get an explicit construction of this involution.

Applying (3.1), we see that the Enriques involution on the Hessian quartic surface associated to a Kummer surface $K$ is given by the linear system defined by the divisor

$$
\eta_{S} \sim \frac{1}{2}\left(3 \eta_{H}-\mathcal{N}\right) \sim 3 \eta_{K}-\frac{3}{2} \sum_{p \in W} E_{p}-\frac{1}{2} \sum_{p \notin W} E_{p},
$$

where $\eta_{K}$ is the class of a hyperplane section of the Kummer surface $K$ in $\mathbb{P}^{3}$, $W$ is the Weber hexad of nodes corresponding to the Weber hexad of 2-torsion points, and $E_{p}$ is the class of the exceptional curve blown up from one of the 16 nodes of $K$. On the abelian surface $J$ this corresponds to the linear system $\left|6 \Theta-3 \sum_{\mu \in h} \mu-\sum_{\mu \notin h} \mu\right|$. The linear system

$$
\left|6 \Theta-3 \sum_{\mu \in h} \mu-\sum_{\mu \notin h} \mu+\left(4 \Theta-2 \sum_{\mu \in h} \mu\right)\right|=\left|10 \Theta-5 \sum_{\mu \in h} \mu-\sum_{\mu \notin h} \mu\right|
$$

maps $J$ onto an Enriques surface embedded in $\mathbb{P}^{5}$ by its Fano linear system of degree 10.

If we choose the Weber hexad (4.3), then the Sylvester pentahedron of the corresponding Hessian quartic is given in Figure 2.

We shall use the previous notation for nodes and lines on a general Hessian surface.

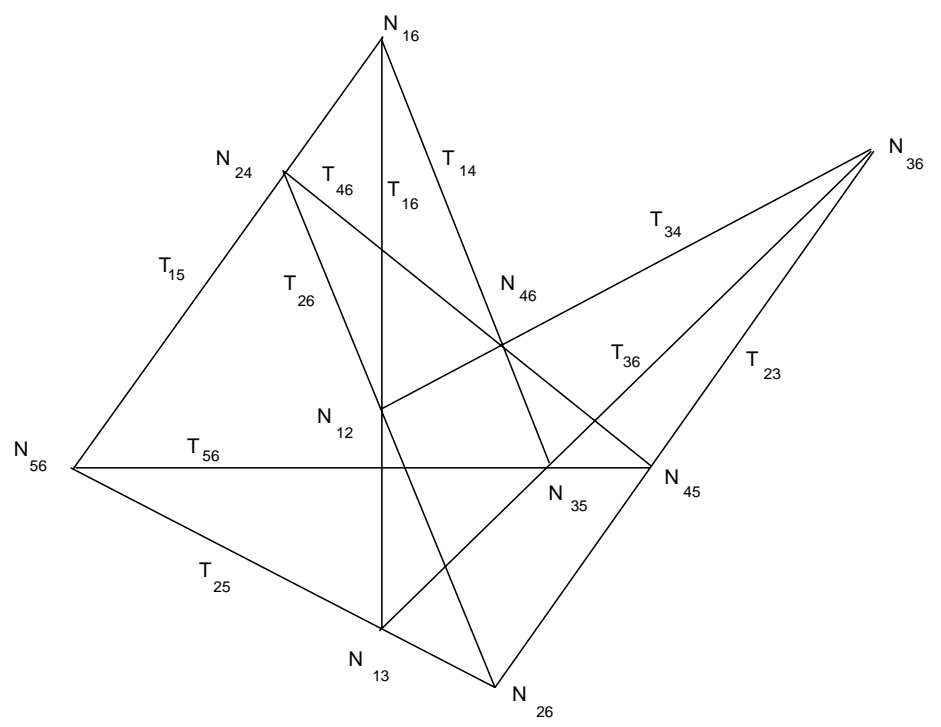

FIGURE 2.

\section{Elliptid PENCILS}

There are many elliptic pencils on the surface $\tilde{H}$. We list a few, which will be used later. We accommodate the notations for the conics $C_{\alpha}$ (3.4) and cubics $R_{\alpha}$ (3.5) to our new notation for $(-2)$-curves $T_{\alpha}$ and $N_{\beta}$. 
Type 1. It is cut out by the linear pencil of planes through an edge of the pentahedron:

$$
\left|F_{\alpha}\right|=\left|C_{\alpha}+T_{\alpha}\right|
$$

We have

$$
F_{\alpha} \cdot F_{\beta}=2, \quad \text { if } T_{\alpha} \text { and } T_{\beta} \text { are skew. }
$$

The pencil $\left|F_{\alpha}\right|$ has 2 reducible fibres of type $I_{2}$ :

$$
C_{\alpha}+T_{\alpha}, \quad N_{\bar{\alpha}}+R_{\bar{\alpha}}
$$

where $R_{\bar{\alpha}}$ is the residual cubic for the plane section of $H$ passing through the edge $T_{\alpha}$ and its opposite node $N_{\bar{\alpha}}$ (see (3.5) ). It has also two reducible fibres of type $I_{6}$ corresponding to the two faces containing $T_{\alpha}$.

Observe that the Enriques involution leaves the pencil invariant by interchanging two degenerate fibres of the same type. The members of the pencil are cubic curves.

The pencil of type 1 is denoted by $F_{i}$ in [CD]. It is the pre-image of one of the ten pencils on the Enriques surface $Y$ as explained in Section 2. Using (3.2), we have

$$
\left|\tilde{\Delta}-2 F_{i}\right|=B_{i}+\tau\left(B_{i}\right)
$$

where $\tau$ is the Enriques involution, and $B_{i}$ is of the form $T_{15}+N_{56}+N_{24}+N_{16}$. This agrees with the notation from [CD].

Type 2. This is a pencil $\left|F_{N_{\alpha}, T_{\beta}}\right|$ on $\tilde{H}$ formed by proper transform of quartic elliptic curves cut out by the pencil of quadric cones with the vertex at a node $N_{\alpha}$ which contain the lines through $N_{\alpha}$ and tangent along one of them, $T_{\beta}$. For example,

$$
\begin{aligned}
& \left|F_{N_{16}, T_{15}}\right| \\
& \quad=\left|2 \eta_{H}-2 T_{15}-T_{16}-T_{14}-N_{24}-N_{56}-N_{12}-N_{13}-N_{46}-N_{35}-2 N_{16}\right| .
\end{aligned}
$$

It has one reducible fibre of type $I_{8}$ :

$$
T_{25}+N_{26}+T_{26}+N_{24}+T_{46}+N_{45}+T_{56}+N_{56},
$$

and two reducible fibres of type $I_{4}$ :

$$
C_{15}+N_{36}+T_{34}+T_{36}, \quad C_{23}+N_{16}+T_{16}+T_{14} .
$$

Observe that $N_{46}, N_{35}, N_{13}, N_{12}$ are sections and $T_{23}, T_{15}$ are bisections of the elliptic fibration. Let us take $N_{46}$ as the zero section. We easily check (by intersecting both sides with any $N_{\alpha}$ and any $T_{\beta}$ ) that

$$
\begin{aligned}
2 N_{13}-2 N_{46} \sim & -T_{36}+T_{34}+T_{14}-T_{16}+T_{46}-N_{56}-2 T_{25} \\
& -N_{26}+N_{24}+2 T_{46}+N_{45} .
\end{aligned}
$$

This implies that $N_{13}$ is a 2-torsion section. Also,

$$
N_{46}+N_{12} \sim N_{13}+N_{35}-N_{46}-T_{34}+T_{36}-T_{46}+T_{56}+N_{56}+T_{25}-T_{26}-N_{24}
$$

implies that $N_{13} \oplus N_{35}=N_{12}$, where $\oplus$ is the group operation on the set of sections with zero section defined by $N_{46}$. One also checks that the translation by $N_{13}$ sends $T_{15}$ to $R_{36}$ and $T_{23}$ to $R_{16}$.

Note that

$$
\left|F_{N_{16}, T_{15}}\right|=\left|F_{N_{36}, T_{23}}\right| .
$$

There are 15 elliptic pencils of type 2 . 
Type 3. This is a pencil $\left|F_{T_{\beta}, T_{\gamma}}\right|$ on $\tilde{H}$ of proper transforms of quartic elliptic curves which are cut out by the pencil of quadric cones with the vertex at a node $N_{\alpha}$ and tangent to $H$ along two edges $T_{\beta}, T_{\gamma}$ intersecting at $N_{\alpha}$. It is spanned by the double plane through the two edges and the union of two planes tangent along these edges. For example,

$$
\begin{gathered}
\left|F_{T_{16}, T_{14}}\right|=\left|2 \eta_{H}-2 T_{16}-2 T_{14}-N_{12}-N_{13}-N_{46}-N_{35}-2 N_{16}\right| \\
=\left|C_{16}+C_{14}\right| .
\end{gathered}
$$

It has reducible fibre of type $I_{0}^{*}$ and $I_{2}^{*}$ :

$$
\begin{gathered}
C_{23}+2 T_{15}+N_{16}+N_{56}+N_{24}, \\
2 T_{36}+2 T_{34}+2 N_{36}+N_{13}+N_{12}+N_{46}+N_{35} .
\end{gathered}
$$

Also it has a reducible fibre of type $I_{2}$ :

$$
C_{16}+C_{14}
$$

We see that $N_{45}$ and $N_{26}$ are contained in fibres. Since $C_{16}+C_{14}-N_{26}$ (resp. $C_{16}+C_{14}-N_{45}$ ) is of degree 4 with respect to $\eta_{H}$, and cannot be a union of two conics, we get two more reducible fibres of type $I_{2}$. Observe that $T_{26}, T_{56}, T_{25}$ and $T_{46}$ are sections and $T_{16}, T_{14}, T_{23}$ are bisections. Let us take $T_{46}$ as the zero section. Then

$$
\begin{aligned}
2 T_{25}-2 T_{46} \sim & 2 T_{36}+3 N_{36}+4 T_{34}+N_{35}+3 N_{46}+2 N_{12}-N_{56} \\
& +N_{24}+C_{16}+N_{45}-N_{26}-2\left(C_{16}+C_{14}\right)
\end{aligned}
$$

implies that $T_{25}$ is a 2-torsion section. Also $T_{25} \oplus T_{56}=T_{26}$.

\section{Birational involutions of a Hessian quartic}

There are some obvious birational involutions of a Hessian quartic surface. They are:

(i) the Enriques involution $\tau$;

(ii) an involution $p_{\alpha}$ defined by projection from a node $N_{\alpha}$;

(iii) an involution defined by a pair of skew lines;

(iv) an involution defined by two elliptic pencils $|F|$ and $\left|F^{\prime}\right|$ with $F \cdot F^{\prime}=2$. This is the covering involution for a degree 2 map $\tilde{H} \rightarrow \mathbb{P}^{1} \times \mathbb{P}^{1}$ defined by the linear system $\left|F+F^{\prime}\right|$.

(v) an involution defined by the inversion map of an elliptic fibration with a section;

(vi) an involution defined by the translation by a 2-torsion section in the group law of sections of an elliptic fibration.

Let us describe the action of each involution on the Picard lattice $S_{H}$ of $\tilde{H}$.

(i) We have already described the action of the Enriques involution. In our new notation we have

$$
\begin{aligned}
& N_{16} \longleftrightarrow T_{23}, N_{24} \longleftrightarrow T_{36}, N_{56} \longleftrightarrow T_{34}, N_{12} \longleftrightarrow T_{56}, N_{13} \longleftrightarrow T_{46}, \\
& N_{26} \longleftrightarrow T_{14}, N_{35} \longleftrightarrow T_{26}, N_{46} \longleftrightarrow T_{25}, N_{36} \longleftrightarrow T_{15}, N_{45} \longleftrightarrow T_{16} .
\end{aligned}
$$

We already know that the image of $\eta_{H}$ is equal to $\eta_{S}$. 
(ii) We may assume that we project to the plane defined by the opposite face of the pentahedron. Then the projection $p_{16}$ from $N_{16}$ acts as follows:

$$
\begin{gathered}
N_{56} \longleftrightarrow N_{24}, N_{13} \longleftrightarrow N_{12}, N_{35} \longleftrightarrow N_{46}, \\
T_{25} \longleftrightarrow T_{26}, T_{36} \longleftrightarrow T_{34}, T_{56} \longleftrightarrow T_{46}, \\
T_{23} \longleftrightarrow \eta_{H}-N_{16}-T_{23}-N_{26}-N_{45}-N_{36}=R_{16},
\end{gathered}
$$

and the remaining curves $N_{26}, N_{36}, N_{45}, T_{15}, T_{16}, T_{14}$ are fixed.

By (3.4) and (3.5), $N_{16}+R_{16}=C_{23}+T_{23}$, and hence

$$
N_{16} \longleftrightarrow C_{23} \text {. }
$$

Since $\eta_{H}=C_{23}+2 T_{23}+N_{36}+N_{45}+N_{26}$, we obtain

$$
\begin{aligned}
\eta_{H} & \longleftrightarrow N_{16}+2 R_{16}+\left(N_{36}+N_{45}+N_{26}\right) \\
& =2 \eta_{H}-2 T_{23}-N_{26}-N_{45}-N_{36}-N_{16} .
\end{aligned}
$$

Thus the involution is given by the linear system of quadrics through the vertex $N_{16}$ and touching $H$ along the edge $T_{23}$.

Projections $p_{\alpha}$ commute with the Enriques involution $\tau$, i.e.

$$
p_{\alpha} \circ \tau=\tau \circ p_{\alpha}
$$

(iii) This is a special case of (iv) when the two pencils are of type 1 and correspond to two skew edges. Assume that the edges are $T_{15}$ and $T_{23}$. Let the fibres $N_{12}+$ $T_{16}+N_{13}+T_{25}+N_{26}+T_{26}$ and $T_{14}+T_{46}+N_{46}+N_{15}+T_{56}+N_{35}$ of $F_{15}$ go to the lines $A_{1}, A_{2}$ on the quadric $\mathbb{P}^{1} \times \mathbb{P}^{1}$. Similarly, let $T_{56}+T_{36}+T_{25}+N_{56}+N_{35}+N_{13}$ and $T_{26}+T_{46}+T_{34}+N_{12}+N_{46}+N_{24}$ go to the lines $B_{1}, B_{2}$ of the other ruling. Then the pre-image of $A_{1}$ splits in $T_{16}+N_{26}$, the pre-image of $A_{2}$ splits in $T_{14}+N_{45}$, the pre-image of $B_{1}$ splits in $T_{36}+N_{56}$, the pre-image of $B_{2}$ splits in $T_{34}+N_{24}$. This easily shows that the action of the involution is defined as follows:

$$
\begin{aligned}
& T_{16} \longleftrightarrow N_{26}, T_{14} \longleftrightarrow N_{45}, T_{36} \longleftrightarrow N_{56}, T_{34} \longleftrightarrow N_{24}, \\
& T_{15} \longleftrightarrow C_{15}, N_{36} \longleftrightarrow R_{36}, N_{16} \longleftrightarrow R_{16}, T_{23} \longleftrightarrow C_{23}, \\
& T_{25} \longleftrightarrow N_{13}, T_{56} \longleftrightarrow N_{35}, T_{26} \longleftrightarrow N_{12}, T_{46} \longleftrightarrow N_{46} .
\end{aligned}
$$

This implies that

$$
\eta_{H} \longleftrightarrow T_{15}+2 C_{15}+T_{36}+T_{34}+R_{16}
$$

This involution is the same as the composition $\tau \circ p_{16} \circ p_{36}$.

(iv) Let us consider the two pencils defined by planes through non-skew edges. Take the edges $T_{15}$ and $T_{25}$. Computations similar to (iii) show that the involution coincides with the projection from $N_{56}$.

(v) Consider a pencil of type 3 with reducible fibres

$$
\begin{array}{ccc}
C_{16}+C_{14}, & N_{26}+N_{26}^{\prime}, \quad N_{45}+N_{45}^{\prime}, \quad C_{23}+2 T_{15}+N_{16}+N_{56}+N_{24}, \\
2 T_{36}+2 T_{34}+2 N_{36}+N_{13}+N_{12}+N_{46}+N_{35} .
\end{array}
$$

We verify that $T_{46}, T_{56}, T_{26}$ and $T_{25}$ are sections. Let us take $T_{46}$ as the zero section. We have already observed that $T_{25}$ is a 2 -torsion section. Consider the automorphism $\phi$ of the surface defined by the inversion map with respect to the group law on the set of sections with zero section defined by $T_{46}$. Obviously, $T_{46}$ and $T_{25}$ are invariant. Also, the components of reducible fibres which they intersect 
are also invariant. Finally, the multiple components of fibres are invariant. This easily shows that all irreducible components of fibres are invariant. Let $M$ be the sublattice spanned by irreducible components of fibres and the sections $T_{46}, T_{25}$. Its rank is 15 . Let $\alpha \in S_{H}$ be a primitive vector orthogonal to $M$. Obviously, $\phi(\alpha)=-\alpha$. One easily finds the vector $\alpha$ :

$$
\begin{aligned}
\alpha= & 2 \eta_{H}-2 \eta_{S}+2\left(T_{46}+T_{25}+T_{34}+T_{36}+T_{15}\right) \\
& +\left(N_{36}+N_{46}+N_{56}+N_{13}+N_{24}\right) .
\end{aligned}
$$

We leave it to the reader to check that $\alpha$ is a $(-6)$-root of $S_{H}$. Therefore, the action of $\phi$ on the Picard lattice $S_{H}$ is a reflection

$$
\phi(x)=x-\frac{2(x \cdot \alpha)}{\alpha^{2}} \alpha .
$$

\section{The LEECH LATtiCE}

We follow the notation and the main ideas from Kondō's paper [Ko]. First we embed the Picard lattice $S_{H}$ of $\tilde{H}$ in the lattice $L=\Lambda \perp U \cong I I_{1,25}$, where $\Lambda$ is the negative definite Leech lattice and $U$ is the hyperbolic plane. We denote each vector $x \in L$ by $(\lambda, m, n)$, where $\lambda \in \Lambda$, and $x=\lambda+m f+n g$, with $f, g$ being the standard generators of $U$. Note that, $r=\left(\lambda, 1,-1-\frac{\langle\lambda, \lambda\rangle}{2}\right)$ satisfies $r^{2}=-2$. Such vectors will be called Leech roots. Recall that $\Lambda$ can be defined as a certain lattice in $\mathbb{R}^{24}=\mathbb{R}^{\mathbb{P}^{1}\left(\mathbb{F}_{23}\right)}$ equipped with the inner product $\langle x, y\rangle=-\frac{x \cdot y}{8}$. For any subset $A$ of $\Omega=\mathbb{P}^{1}\left(\mathbb{F}_{23}\right)$ let $\nu_{A}$ denote the vector $\sum_{i \in A} e_{i}$, where $\left\{e_{\infty}, e_{0}, \ldots, e_{22}\right\}$ is the standard basis in $\mathbb{R}^{24}$. Then $\Lambda$ is defined as a lattice generated by the vectors $\nu_{\Omega}-4 \nu_{\infty}$ and $2 \nu_{K}$, where $K$ belongs to the $\operatorname{SL}\left(2, \mathbb{F}_{23}\right)$-orbit of the ordered subset $(\infty, 0,1,3,12,15,21,22)$ of $\Omega$. These sets form a Steiner system $S(5,8,24)$ of eightelement subsets of $\Omega$ such that any five-element subset belongs to a unique element of $S(5,8,24)$. All such sets are explicitly listed in [To].

7.1. Lemma. There is a primitive embedding of $S_{H}$ in $L$ such that $S_{H}^{\perp}$ contains a sublattice of index 2 isomorphic to the root lattice $R=A_{5}+A_{1}^{5}$.

Proof. Consider the following Leech roots:

$$
\begin{gathered}
x=\left(4 \nu_{\infty}+\nu_{\Omega}, 1,2\right), \quad y=\left(4 \nu_{0}+\nu_{\Omega}, 1,2\right), \quad z=(0,1,-1), \\
x_{0}=\left(4 \nu_{\infty}+4 \nu_{0}, 1,1\right), \quad x_{i}=\left(2 \nu_{K_{i}}, 1,1\right), \quad i=1, \ldots, 5,
\end{gathered}
$$

where

$$
\begin{gathered}
K_{1}=\{\infty, 0,1,2,3,5,14,17\}, \\
K_{2}=\{\infty, 0,1,2,4,13,16,22\}, \quad K_{3}=\{\infty, 0,1,2,6,7,19,21\}, \\
K_{4}=\{\infty, 0,1,2,8,11,12,18\}, \quad K_{5}=\{\infty, 0,1,2,9,10,15,20\} .
\end{gathered}
$$

It is easy to verify that the inner product of the vectors $x, y, z, x_{i}$ is described by the following (reducible) Coxeter-Dynkin diagram:

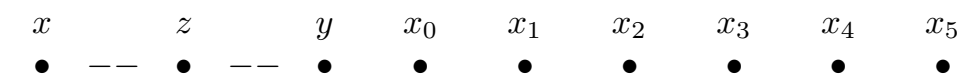


Thus these vectors span a root sublattice $R_{0}$ of $L$ isomorphic to $A_{3}+A_{1}^{6}$. We shall add one more vector to the previous set. Let

$$
r_{0}=\left(2 \nu_{K_{0}}, 1,1\right), \quad K_{0}=\{\infty, 1,2,3,4,6,15,18\} .
$$

One verifies that

$$
\begin{gathered}
\left\langle r_{0}, y\right\rangle=\left\langle r_{0}, x_{0}\right\rangle=1, \\
\left\langle r_{0}, x\right\rangle=\left\langle r_{0}, z\right\rangle=\left\langle r_{0}, x_{i}\right\rangle=0, \quad i \neq 0 .
\end{gathered}
$$

Thus the new Dynkin diagram looks like

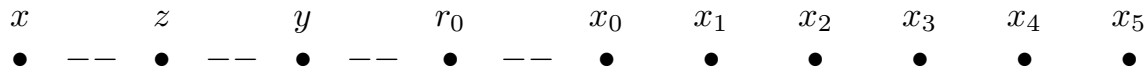

So, the new lattice $R$ spanned by $x, y, z, x_{i}, r_{0}$ is isomorphic to $A_{5}+A_{1}^{5}$. Let

$$
\theta=\frac{1}{2}\left(x+y+\sum_{i=0}^{5} x_{i}\right)
$$

Let $q_{M}: D(M)=M^{*} / M \rightarrow \mathbb{Q} / 2 \mathbb{Z}$ denote the discriminant quadratic form of a lattice $M$. We have

$$
\begin{gathered}
D\left(A_{5}\right)=\left(\frac{5 x+4 z+3 y+2 r_{0}+x_{0}}{6}\right), \quad q_{A_{5}}=\left\langle-\frac{5}{6}\right\rangle, \\
D\left(A_{1}\right)=\left(\frac{x_{i}}{2}\right), \quad q_{A_{1}}=\left\langle-\frac{1}{2}\right\rangle .
\end{gathered}
$$

Write $\bar{\theta}=\theta \in D(R)$ as a sum of $v=\frac{5 x+4 z+3 y+2 r+x_{0}}{6}$ and $\beta_{i}=\frac{x_{i}}{2}$ modulo $R$. We have $\bar{\theta}=3 v+\sum_{i=1}^{5} \beta_{i}$. It is checked that $\bar{\theta}$ spans a subgroup $A$ of $D(R)$ which is isotropic with respect to the discriminant quadratic form $q_{R}$. Let $T$ be the overlattice of $R$ corresponding to the group $A^{\perp} / A$. It is easy to check that

$$
\begin{gathered}
q_{T}=q_{R} \mid A^{\perp} / A=\left\langle v+\beta_{1}, v+\beta_{2}, v+\beta_{3}, v+\beta_{4}, v+\beta_{5}\right\rangle / \bar{\theta} \\
=\left\langle v+\beta_{1}+\beta_{3}+\beta_{5}, \beta_{1}+\beta_{2}\right\rangle \oplus\left\langle\beta_{1}+\beta_{2}+\beta_{3}+\beta_{4}, \beta_{1}+\beta_{2}+\beta_{4}+\beta_{5}\right\rangle \\
=q_{A_{2}(2)} \oplus q_{U(2)} .
\end{gathered}
$$

Thus $T=\langle R, \theta\rangle$ has the same discriminant quadratic form as the transcendental lattice $T_{\tilde{H}}$. We skip the verification that $T$ is primitive. It is similar to the proof of Lemma 4.1 from $[\mathrm{Ko}$. Thus the orthogonal complement of $T$ in $L$ is a primitive lattice of rank 16 with the same discriminant form as the Picard lattice $S_{H}$. Now the result follows from the uniqueness theorem of Nikulin [Ni].

7.2. Remark. Note that the orthogonal complement of the lattice $R_{0}$ in $L$ is isomorphic to the Picard lattice $S_{K}$ of a general Jacobian Kummer surface. It contains the sublattice $S_{H}$ as the orthogonal complement of the projection of $r_{0}$ in $S_{K}$.

One can give an explicit formula for twenty vectors $N_{\alpha}^{\prime}, T_{\beta}^{\prime} \in T^{\perp}$ whose intersection matrix coincides with the intersection matrix of the divisor classes $N_{\alpha}, T_{\beta}$. We shall identify $N_{\alpha}^{\prime}, T_{\beta}^{\prime}$ with $N_{\alpha}, T_{\beta}$.

Explicitly, $N_{\alpha}, T_{\beta}$ correspond to the Leech roots $\left(2 \nu_{K}, 1,1\right)$, where $K \subset \Omega$ is

$$
\begin{aligned}
& N_{45}:\{\infty, 0,1,3,4,11,19,20\}, \quad N_{56}:\{\infty, 0,1,3,6,8,10,13\}, \\
& N_{24}:\{\infty, 0,1,3,7,9,16,18\}, \quad N_{26}:\{\infty, 0,1,3,12,15,21,22\},
\end{aligned}
$$




$$
\begin{gathered}
\text { Birational automorphisms of Quartic Hessian surfaces } \\
N_{36}:\{\infty, 0,1,4,5,7,8,15\}, \quad N_{35}:\{\infty, 0,1,4,6,9,12,17\}, \\
N_{46}:\{\infty, 0,1,4,10,14,18,21\}, \quad N_{16}:\{\infty, 0,1,5,6,18,20,22\}, \\
N_{13}:\{\infty, 0,1,6,11,14,15,16\}, \quad N_{12}:\{\infty, 0,1,13,15,17,18,19\}, \\
T_{16}:\{\infty, 0,2,3,4,8,9,21\}, \quad T_{34}:\{\infty, 0,2,3,6,12,16,20\}, \\
T_{14}:\{\infty, 0,2,3,7,11,13,15\}, \quad T_{36}:\{\infty, 0,2,3,10,18,19,22\}, \\
T_{26}:\{\infty, 0,2,4,5,6,10,11\}, \quad T_{25}:\{\infty, 0,2,4,7,17,18,20\}, \\
T_{15}:\{\infty, 0,2,4,12,14,15,19\}, \quad T_{56}:\{\infty, 0,2,5,15,16,18,21\}, \\
T_{46}:\{\infty, 0,2,6,8,15,17,22\}, \quad T_{23}:\{\infty, 0,2,6,9,13,14,18\} .
\end{gathered}
$$

For Leech roots $r, r^{\prime} \in L$ corresponding to the Leech vectors $\lambda, \lambda^{\prime}$ we have

$$
\left(r, r^{\prime}\right)= \begin{cases}0, & \text { if } \lambda-\lambda^{\prime} \in \Lambda_{4} \\ 1, & \text { if } \lambda-\lambda^{\prime} \in \Lambda_{6}\end{cases}
$$

Here

$$
\begin{gathered}
\Lambda_{4}=\{x \in \Lambda:(x, x)=4\}=\left\{\left( \pm 2^{8}, 0^{16}\right),\left( \pm 3, \pm 1^{23}\right),\left( \pm 4^{2}, 0^{22}\right)\right\} \\
\Lambda_{6}=\{x \in \Lambda:(x, x)=6\} \\
=\left\{\left( \pm 2^{12}, 0^{12}\right),\left( \pm 3^{3}, \pm 1^{21}\right),\left( \pm 4, \pm 2^{8}, 0^{15}\right),\left( \pm 5, \pm 1^{23}\right)\right\}
\end{gathered}
$$

Let

$$
\omega=(0,0,1) \in L
$$

It is called the Weyl vector of the lattice $L$. It is an isotropic vector with the property that

$$
(\omega, l)=1 \quad \text { for any Leech root } l
$$

7.3. Lemma. The projection $\omega^{\prime}$ of $\omega$ in $S_{H}$ is equal to the vector

$$
\tilde{\Delta}=\mathcal{N}+\mathcal{T}
$$

Proof. Note that $\left(\omega^{\prime}, N_{\alpha}\right)=\left(\omega^{\prime}, T_{\beta}\right)=1$ for all $\alpha, \beta$. On the other hand, the divisor $\mathcal{N}+\mathcal{T}=\sum N_{\alpha}+\sum T_{\beta}$ has the same property. The result follows from Corollary 4.2 . 


\section{Automorphisms of a general Hessian quartic Surface}

Let $X$ be a K3 surface with Picard lattice $S$. The automorphism group $\operatorname{Aut}(X)$ of $X$ has a natural representation $\rho: \operatorname{Aut}(X) \rightarrow O(S)$ in the orthogonal group of $S$. Let $W_{2}(S)$ denote the subgroup generated by reflections in vectors $r$ with $r^{2}=-2$. This group is a normal subgroup of $O(X)$, and the induced homomorphism

$$
\rho: \operatorname{Aut}(X) \rightarrow O(S) / W_{2}(S)
$$

has a finite kernel and a finite cokernel. This non-trivial result follows from the Global Torelli Theorem for algebraic K3 surfaces proven by I. Piatetski-Shapiro and I. Shafarevich [PS]. Let us describe the kernel and the cokernel. First of all, the group $O(S) / W_{2}(S)$ has the following interpretation. Let $V_{S}=\{x \in S \otimes \mathbb{R}$ : $\left.x^{2}>0\right\}$, and let $V_{S}^{+}$be its connected component containing an ample divisor class. The group $W_{2}(S)$ has a fundamental domain $P(S)$ in $V_{S}^{+}$(a cone over a convex polytope in the corresponding Lobachevski space $\left.V_{S}^{+} / \mathbb{R}_{+}\right)$. It can be chosen in such a way that its bounding hyperplanes are orthogonal to effective classes $r$ with $r^{2}=-2$ and it contains the ray spanned by an ample divisor class. Let $A(P(S)) \subset O(S)$ be the group of symmetries of $P(S)$. Then $O(S)$ is equal to the semi-direct product $W_{2}(S) \rtimes A(P(S))$ of $W_{2}(S)$ and $A(P(S))$. The image of Aut $(X)$ in $O(S)$ is contained in $A(P(S))$. Let $D(S)=S^{*} / S$ be the discriminant group of the lattice, and $q_{S}$ the discriminant quadratic form on $D(S)$. We have a natural homomorphism $A(P(S)) \rightarrow O\left(q_{S}\right)$. Let $\Gamma(S) \subset A(P(S))$ be the pre-image of $\{ \pm 1\}$. Then the image of $\operatorname{Aut}(X)$ in $A(P(S))$ is contained in $\Gamma(S)$ as a subgroup of index $\leq 2$. It is equal to the whole group $\Gamma(S)$ if $\operatorname{Aut}(X)$ contains an element acting as -1 on $D(S)$. This will be the case for the Hessian, surface. The kernel of $\operatorname{Aut}(X) \rightarrow \Gamma(S)$ is a finite cyclic group. It is trivial if $X$ does not admit a nontrivial automorphism preserving any ample divisor. This happens in our case for a general Hessian, since it is known that a projective automorphism of a general cubic surface is trivial. Summing up, we obtain

8.1. Proposition. Let $S_{H}$ be the Picard lattice of a minimal nonsingular model $\tilde{H}$ of a general Hessian quartic surface $H$. The group of automorphisms of $\tilde{H}$ is isomorphic to the group $\Gamma\left(S_{H}\right)$ of symmetries of $P\left(S_{H}\right)$ which act as \pm 1 on the discriminant group of $S_{H}$.

Let $W_{\text {Lee }}(L)$ be the subgroup of $O(L)$ generated by reflections in Leech roots. Let $P(L)$ be its fundamental domain in the $V_{L}^{+}$(where $V_{L}^{+}$is one of the components of $V=\left\{x \in L \otimes \mathbb{R}: x^{2}>0\right\}$ ) whose closure contains the Weyl vector $\omega$. By a result of J. Conway, $O(L)$ is equal to the semi-direct product $W_{\text {Lee }}(L) \rtimes A(P(L))$ of $W_{\text {Lee }}(L)$ and the group of symmetries $A(P(L))$ of $P(L)$, and the latter is isomorphic to the group $\Lambda \rtimes O(\Lambda)$ of affine automorphisms of $\Lambda$.

Now put

$$
P\left(S_{H}\right)^{\prime}=P(L) \cap V\left(S_{H}\right)^{+}
$$

It is known that $P\left(S_{H}\right)^{\prime}$ is non-empty, has only finitely many faces, and is of finite volume (in the Lobachevski space). Also it is known ([Bo], Lemmas 4.1-4.3) that the projection $\omega^{\prime}$ of the Weyl vector is contained in $P\left(S_{H}\right)^{\prime}$. Applying Lemma 8.3, we see that $P\left(S_{H}\right)^{\prime}$ contains an ample divisor class, and hence $P\left(S_{H}\right)^{\prime}$ is a part of $P\left(S_{H}\right)$. 
8.2. Lemma. Let $G=\operatorname{Aut}\left(P\left(S_{H}\right)^{\prime}\right)$ be the group of symmetries of $P\left(S_{H}\right)^{\prime}$. Then $G$ is isomorphic to $\mathbb{Z} / 2 \times S_{5}$ and can be realized as the subgroup of $O\left(S_{H}\right)$ generated by the Enriques involution and the group of symmetries of the Sylvester pentahedron. It extends to a subgroup of $O(L)$ which leaves the root lattice $R$ invariant and induces the isometries of $R$ defined by the symmetries of the Dynkin diagram of $R$.

Proof. This is almost a word-for-word repetition of the proof of Lemma 4.5 in Ko.

To find a certain set of generators of $\operatorname{Aut}(\tilde{H})$ containing the Enriques involution, we use the following strategy suggested in [Ko]. First we shall find the hyperplanes which bound $P\left(S_{H}\right)^{\prime}$. They correspond to rank 11 root sublattices $R^{\prime}$ generated by $R$ and some Leech root $r$. The hyperplane $\left\{x \in V_{L}^{+}:(x, r)=0\right\}$ is a boundary wall of $P(L)$ and has non-empty intersection with $P\left(S_{H}\right)$. Then for such a hyperplane we find an automorphism of $\tilde{H}$ which maps one of the two half-spaces defined by this hyperplane to the opposite one. Let $N$ be the group generated by these automorphisms. Then we check that for any automorphism $\gamma \in \operatorname{Aut}(\tilde{H})$ one can find $\delta \in N$ such that $\delta \circ \gamma$ is a symmetry of $P\left(S_{H}\right)^{\prime}$. Applying Lemma 9.2, we conclude that $\delta \circ \gamma$ is either the identity or the Enriques involution (since the latter and the identity are the only elements of $\operatorname{Aut}\left(P\left(S_{H}\right)^{\prime}\right)$ which act as \pm 1 on the discriminant group of $S_{H}$ ).

The next lemma is a simple repetition of the computations from [Ko], Lemma 4.6 .

8.3. Lemma. Let $r$ be a Leech root. Assume that $r$ and $R$ generate a root lattice $R^{\prime}$ of rank 11. Then one of the following cases occurs:

(0) $R^{\prime}=A_{5} \oplus A_{1}^{6}$, where $r$ is orthogonal to $R$;

(1a) $R^{\prime}=D_{6} \oplus A_{1}^{5}$, where $\left(r, r_{0}\right)=0,(r, z)=1$;

(1b) $R^{\prime}=D_{6} \oplus A_{1}^{5}$, where $\left(r, r_{0}\right)=1,(r, z)=0$;

(2) $R^{\prime}=A_{1}^{3} \oplus A_{3} \oplus A_{5}$, where $\left(r, x_{i}\right)=\left(r, x_{j}\right)=1, i, j \neq 0$;

(3a) $R^{\prime}=A_{7} \oplus A_{1}^{4}$, where $\left(r, x_{0}\right)=\left(r, x_{i}\right)=1$;

(3b) $R^{\prime}=A_{7} \oplus A_{1}^{4}$, where $(r, x)=\left(r, x_{i}\right)=1$.

Moreover, in case (0), $r$ is one of the twenty Leech roots corresponding to $N_{\alpha}$ and $T_{\beta}$.

In case (1a), up to a symmetry of $P\left(S_{H}\right)^{\prime}$, we can choose

$$
r=\left(\lambda, 1,-1-\frac{\langle\lambda, \lambda\rangle}{2}\right)
$$

corresponding to the Leech vector

$$
\begin{aligned}
\lambda & =\left(\xi_{\infty}, \xi_{0}, \xi_{j_{1}}, \xi_{j_{2}}, \ldots, \xi_{j_{6}}, \xi_{j_{7}}, \ldots, \xi_{j_{22}}\right) \\
& =(3,3,3,-1, \ldots,-1,1, \ldots, 1),
\end{aligned}
$$

where $K=\left\{\infty, 0, j_{1}, \ldots, j_{6}\right\}$ is an octad satisfying

$$
\left|K \cap K_{0}\right|=\left\{\infty, j_{1}\right\}, \quad\left|K \cap K_{i}\right|=4
$$

and $j_{1} \in K_{i}$ for $i=1, \ldots, 5$.

In case (1b), $r$ corresponds to $N_{\alpha}, \alpha \in\{0,14,15,23,25,34\}$, or to $T_{\beta}, \beta \in\{0,12$, $13,24,35,45\}$. Together with "old" $N_{\alpha}$ and $T_{\beta}$ they define 32 vectors spanning the Kummer overlattice $S_{K}$ of $S_{H}$. 
In case (2), $r$ corresponds to a Leech vector

$$
\begin{gathered}
\lambda=2 \nu_{K}, \quad 0, \infty \in K, \quad\left|K \cap K_{i}\right|=\left|K \cap K_{j}\right|=2, \\
\left|K \cap K_{l}\right|=4 \quad \text { for } l=0, \ldots, 5, l \neq i, j .
\end{gathered}
$$

In case (3a), if $r$ meets $x_{0}, x_{i}$, it corresponds to a Leech vector

$$
\lambda=\nu_{\Omega}-4 \nu_{k}, \quad k \neq 0, \infty, k \in K_{i}, k \notin K_{j}, j=0,1, \ldots, 5, j \neq i .
$$

In case (3b), if $r$ meets $x, x_{i}$, it corresponds to a Leech vector

$$
\begin{gathered}
\lambda=4 \nu_{0}-\nu_{K}+\nu_{\Omega-K}, \quad 0 \in K, \infty \notin K, \\
\left|K \cap K_{0}\right|=0, \quad\left|K \cap K_{i}\right|=4, \quad\left|K \cap K_{l}\right|=2, \quad l=1, \ldots, 5, l \neq i .
\end{gathered}
$$

8.4. Remark. The number of vectors $r$ in case (1a) is equal to 12. They correspond to the following octads $K$ :

$$
\begin{array}{r}
\{\infty, 0,1,5,9,11,13,21\}, \quad\{\infty, 0,1,7,10,11,17,22\}, \\
\{\infty, 0,1,7,12,13,14,20\}, \quad\{\infty, 0,1,8,9,14,19,22\}, \\
\{\infty, 0,1,8,16,17,20,21\}, \quad\{\infty, 0,1,5,10,12,16,19\}, \\
\{\infty, 0,2,9,11,16,17,19\}, \quad\{\infty, 0,2,5,8,13,19,20\}, \\
\{\infty, 0,2,7,8,10,14,16\}, \quad\{\infty, 0,2,5,7,9,12,22\}, \\
\{\infty, 0,2,10,12,13,17,21\}, \quad\{\infty, 0,2,11,14,20,21,22\} .
\end{array}
$$

The number of vectors $r$ in case (2) is equal to $10=\left(\begin{array}{l}5 \\ 2\end{array}\right)$. When $i=1, j=2, r$ corresponds to the following octad $K$ :

$$
\{0, \infty, 6,7,10,12,15,18\} .
$$

The number of vectors $r$ in case (3a) is equal to $5 \cdot 3=15$. When $i=1$, they correspond to the following Leech vectors:

$$
\nu_{\Omega}-4 \nu_{k}, \quad k \in\{5,14,17\} .
$$

The number of vectors $r$ in case (3b) is equal to $5 \cdot 3=15$. When $i=1$, they correspond to the following octads $K$ :

$$
\{0,5,9,12,13,14,17,19\}, \quad\{0,5,10,11,14,16,17,21\},
$$

$$
\{0,5,7,8,14,17,20,22\}
$$


8.5. Lemma. Let $r$ be a Leech root as in the previous lemma. Let $r=r_{1}+r_{2}$, where $r_{1} \in S_{H}^{*}$ and $r_{2} \in T^{*}$. Then

Case (0): $r_{1}=r$;

Case $(1 a): r_{1}=r+\frac{1}{3}\left(2 x+4 z+3 y+2 r_{0}+x_{0}\right),\left(r_{1}, r_{1}\right)=-2 / 3$;

Case $(1 b): r_{1}=r+\frac{1}{3}\left(x+2 z+3 y+4 r_{0}+2 x_{0}\right),\left(r_{1}, r_{1}\right)=-2 / 3$;

Case (2): $r_{1}=r+\frac{1}{2}\left(x_{i}+x_{j}\right),\left(r_{1}, r_{1}\right)=-1$;

Case $(3 a): r_{1}=r+\frac{1}{6}\left(x+2 z+3 y+4 r_{0}+5 x_{0}\right)+\frac{1}{2} x_{i},\left(r_{1}, r_{1}\right)=-2 / 3$;

Case $(3 b): r_{1}=r+\frac{1}{6}\left(5 x+4 z+3 y+2 r_{0}+x_{0}\right)+\frac{1}{2} x_{i},\left(r_{1}, r_{1}\right)=-2 / 3$.

Proof. Case (0) is obvious.

Case (1a): Let $x^{*}, y^{*}, z^{*}, r_{0}^{*}, x_{i}^{*}$ denote the dual basis of the basis $x, y, z, r_{0}, x_{0}$, $\ldots, x_{5}$. Since $(r, z)=1,(r, x)=(r, y)=\left(r, r_{0}\right)=\left(r, x_{i}\right)=0$, we see that

$$
r_{2}=z^{*}=-\left(2 x+4 z+3 y+2 r_{0}+x_{0}\right) / 3
$$

and hence

$$
\left(r_{1}, r_{1}\right)=\left(r-r_{2}, r-r_{2}\right)=(r, r)-\left(r_{2}, r_{2}\right)=-2+4 / 3=-2 / 3 .
$$

Case (1b) Similar to the previous case.

Case (2): Here $r_{2}=x_{i}^{*}+x_{j}^{*}=-\left(x_{i}+x_{j}\right) / 2$. This gives $\left(r_{1}, r_{1}\right)=-1$.

Case (3a): $r_{2}=x_{0}^{*}+x_{i}^{*}=-\frac{1}{6}\left(x+2 z+3 y+4 r_{0}+5 x_{0}\right)-\frac{1}{2} x_{i}$. This gives $\left(r_{1}, r_{1}\right)=-2 / 3$.

Case (3b) Similar to the previous case.

Let $s_{r}: v \rightarrow v+(v, r) r$ be the reflection of $L$ in a Leech root $r$. Then the restriction of $s_{r}$ to $S_{H} \otimes \mathbb{Q}$ is a reflection

$$
s_{r_{1}}(v)=v+\left(v, r_{1}\right) r_{1}
$$

where $r_{1}$ is the projection of $r$ onto $S_{H} \otimes \mathbb{Q}$. This is, in general, not an isometry of $S_{H}$.

To find an automorphism of $\tilde{H}$ corresponding to each vector in case (1a)-(3b), we need to express $r_{1}$ in terms of $N_{\alpha}, T_{\beta}$.

Case (1a). Let $r$ correspond to the Leech vector

$$
3 \nu_{0}+3 \nu_{\infty}+3 \nu_{1}-\nu_{5}-\nu_{9}-\nu_{11}-\nu_{13}-\nu_{21}+\nu_{\Omega-K},
$$

where $K=\{0,1, \infty, 5,9,11,13,21\}$. It is immediately verified that

$$
\begin{gathered}
\left(r, N_{\alpha}\right)=0, \quad \text { for all } \alpha, \\
\left(r, T_{\beta}\right)= \begin{cases}1, & \text { if } \beta=16,26,56,14,23 ; \\
0, & \text { otherwise }\end{cases}
\end{gathered}
$$

This determines $r_{1}$ in the form of a linear combination of $N_{\alpha}, T_{\beta}$. Writing down the corresponding system of linear equations and solving it, we obtain

$$
\begin{aligned}
r_{1}= & \frac{1}{15}\left(-2 \mathcal{T}+2 \mathcal{N}+10\left(T_{36}+T_{46}+T_{15}+T_{25}+T_{34}\right)\right. \\
& \left.+5\left(N_{36}+N_{46}+N_{56}+N_{13}+N_{24}\right)\right) .
\end{aligned}
$$

Let

$$
\alpha=3 r_{1} .
$$


By (3.1), $\mathcal{N}-\mathcal{T}=5 \eta_{H}-5 \eta_{S}$, so that

$$
\begin{aligned}
\alpha= & 2 \eta_{H}-2 \eta_{S}+2\left(T_{36}+T_{46}+T_{15}+T_{25}+T_{34}\right) \\
& +\left(N_{36}+N_{46}+N_{56}+N_{13}+N_{24}\right) .
\end{aligned}
$$

It is easy to check that $\alpha$ is a primitive vector of $S_{H}$ and a $(-6)$-root.

Case (1b). These are conjugate to those in case (1a) by the Enriques involution $\tau$, which can be viewed as an automorphism of $P\left(S_{H}\right)^{\prime}$. For example, take $r=T_{12}$. We know that $\left(r_{1}, T_{\alpha}\right)=0$ for all $\alpha$, and

$$
\left(r_{1}, N_{\beta}\right)= \begin{cases}1, & \text { if } \beta=16,26,12,35,45 ; \\ 0, & \text { otherwise. }\end{cases}
$$

Applying the Enriques involution $\tau$, we see that $\left(\tau\left(r_{1}\right), N_{\alpha}\right)=0$ for all $\alpha$, and

$$
\left(\tau\left(r_{1}\right), T_{\beta}\right)= \begin{cases}1, & \text { if } \beta=16,26,56,14,23 \\ 0, & \text { otherwise }\end{cases}
$$

Thus, $\tau\left(r_{1}\right)$ is nothing but the vector that appeared in Case (1a) above.

Case (2). Let $r=\left(2 \nu_{K}, 1,1\right)$, where $K=\{0, \infty, 6,7,10,12,15,18\}$. We have

$$
\begin{aligned}
& \left(r, N_{\alpha}\right)= \begin{cases}1, & \text { if } \alpha=45 ; \\
0, & \text { otherwise }\end{cases} \\
& \left(r, T_{\beta}\right)= \begin{cases}1, & \text { if } \beta=16 ; \\
0, & \text { otherwise }\end{cases}
\end{aligned}
$$

Notice that $N_{45}$ is the vertex opposite to the edge $T_{16}$. It is easy to see that

$$
\left(r, N_{\alpha}\right)=\left(\frac{1}{2}\left(C_{16}-N_{45}\right), N_{\alpha}\right), \quad\left(r, T_{\beta}\right)=\left(\frac{1}{2}\left(C_{16}-N_{45}\right), T_{\beta}\right)
$$

for all $\alpha$ and $\beta$. Thus

$$
r_{1}=\frac{1}{2}\left(C_{16}-N_{45}\right)=\frac{1}{2}\left(\eta_{H}-2 T_{16}-N_{16}-N_{12}-N_{13}-N_{45}\right) .
$$

Note that $\alpha=2 r_{1}$ is a $(-4)$-root of $S_{H}$. Also note that $\tau(\alpha)=\alpha$.

Case (3a). Let $r$ correspond to the Leech vector $\nu_{\Omega}-4 \nu_{5}$. We have

$$
\begin{aligned}
& \left(r, N_{\alpha}\right)= \begin{cases}1, & \text { if } \alpha=16,36 ; \\
0, & \text { otherwise },\end{cases} \\
& \left(r, T_{\beta}\right)= \begin{cases}1, & \text { if } \beta=26,56 ; \\
0, & \text { otherwise }\end{cases}
\end{aligned}
$$

By solving a system of linear equations we find that

$$
\begin{aligned}
r_{1}= & \frac{1}{2}\left(N_{26}+N_{45}+N_{56}+N_{24}\right)-\frac{1}{3}\left(N_{16}+N_{36}\right) \\
& +\frac{1}{3}\left(N_{13}+N_{46}\right)+\frac{2}{3}\left(T_{46}+T_{25}\right)+\frac{1}{3}\left(T_{15}+T_{23}\right) .
\end{aligned}
$$

It can be checked that the minimum positive integer $k$ with $k r_{1} \in S_{H}$ is 6 , so that

$$
\begin{gathered}
6 r_{1}=3\left(N_{26}+N_{45}+N_{56}+N_{24}\right)-2\left(N_{16}+N_{36}\right)+2\left(N_{13}+N_{46}\right) \\
+4\left(T_{46}+T_{25}\right)+2\left(T_{15}+T_{23}\right)
\end{gathered}
$$


is a primitive $(-24)$-vector in $S_{H}$. We remark that $\alpha=6 r_{1}$ is NOT a root.

Case (3b). These are conjugate to those in case (3a) by the Enriques involution $\tau$. For example, let $r$ correspond to the Leech vector

$$
4 \nu_{0}-\nu_{K}+\nu_{\Omega-K}
$$

where $K=\{0,5,9,12,13,14,17,19\}$. We have

$$
\begin{aligned}
& \left(r, N_{\alpha}\right)= \begin{cases}1 & \text { if } \alpha=12,35 ; \\
0 & \text { otherwise }\end{cases} \\
& \left(r, T_{\beta}\right)= \begin{cases}1, & \text { if } \beta=15,23 ; \\
0 & \text { otherwise }\end{cases}
\end{aligned}
$$

Applying $\tau$, we see that

$$
\begin{aligned}
& \left(\tau\left(r_{1}\right), T_{\alpha}\right)= \begin{cases}1, & \text { if } \alpha=26,56 \\
0, & \text { otherwise }\end{cases} \\
& \left(\tau\left(r_{1}\right), N_{\beta}\right)= \begin{cases}1, & \text { if } \beta=16,36 \\
0 & \text { otherwise }\end{cases}
\end{aligned}
$$

and that $\tau\left(r_{1}\right)$ is the one that appeared in Case (3a) above.

Let us find an involution $\sigma$ of $\tilde{H}$ corresponding to a hyperplane defined by a Leech root $r$ of type (1a), (2), or (3a). If $r_{1}$ denotes the projection of $r$ to $S_{H}$, we need that

$$
\sigma\left(r_{1}\right)=-r_{1}
$$

Automorphisms of type (1a). This is the inversion of an elliptic pencil of type 3. For example, the involution $\phi$ from (6.1) corresponds to the vector $r_{1}$ from (8.1). Since $\alpha=3 r_{1}$ is a $(-6)$-root, we get $\phi\left(r_{1}\right)=-r_{1}$. Also

$$
\phi\left(\omega^{\prime}\right)=\omega^{\prime}+3\left(\omega^{\prime}, r_{1}\right) r_{1}=\omega^{\prime}+15 r_{1},
$$

where $\omega^{\prime}=\sum_{\alpha} N_{\alpha}+\sum_{\beta} T_{\beta}$ is the projection of the Weyl vector (see Lemma 8.3).

Let us explain why the number of such automorphisms is 12 . The inversion of the same elliptic fibration with respect to the different zero section $T_{26}$ (and a 2torsion $T_{56}$ ) gives another involution. Since there are 30 elliptic fibrations of type 3 , we get in this way 60 involutions, but only 12 of them are different.

Automorphisms of type (1b). This is the conjugate involution $\tau \circ \phi \circ \tau$.

Automorphisms of type (2). These are the 10 projections $p_{\alpha}$ from a node $N_{\alpha}$. For example, consider $p_{45}$, whose action can be computed as in Section 7-(ii), and take $r_{1}$ computed in (8.2). It is easy to check that $p_{45}\left(r_{1}\right)=-r_{1}$. Note that $p_{45}$ acts nontrivially on the hyperplane $r_{1}^{\perp}$ in $V\left(S_{H}\right)^{+}$. In other words, $p_{45}$ is not a reflection, but works like a reflection. Also observe that

$$
p_{45}\left(\omega^{\prime}\right)=\omega^{\prime}+4 r_{1}=\omega^{\prime}+2\left(\omega^{\prime}, r_{1}\right) r_{1} .
$$


Automorphisms of type (3a). This is the inversion of an elliptic pencil of type 2. To see this, take $r_{1}$ computed in (8.3).

Consider the elliptic fibration

$$
\begin{aligned}
\left|F_{N_{12}, T_{26}}\right| & =\left|F_{N_{35}, T_{56}}\right|=\left|T_{23}+N_{26}+T_{25}+N_{56}+T_{15}+N_{24}+T_{46}+N_{45}\right| \\
& =\left|C_{56}+T_{34}+N_{12}+T_{16}\right|=\left|C_{26}+T_{36}+N_{35}+T_{14}\right| .
\end{aligned}
$$

Observe that $N_{13}, N_{46}, N_{16}, N_{36}$ are sections. Take $N_{13}$ as the zero section. Then $N_{46}$ is a 2 -torsion. Let $f_{r}=f_{N_{16}, N_{36}, T_{26}, T_{56}}$ be the automorphism of the surface $\tilde{H}$ defined by the inversion map with respect to the group law on the set of sections with zero section defined by $N_{13}$. Take

$$
\begin{aligned}
& D_{1}=\left(N_{26}+N_{45}\right)+2\left(N_{56}+N_{24}\right)-N_{36}+\left(N_{13}+N_{46}\right)+2\left(T_{46}+T_{25}+T_{15}\right), \\
& D_{3}=2\left(N_{26}+N_{45}\right)+\left(N_{56}+N_{24}\right)-N_{16}+\left(N_{13}+N_{46}\right)+2\left(T_{46}+T_{25}+T_{23}\right) .
\end{aligned}
$$

Then $D_{1}$ and $D_{3}$ are effective $(-2)$-vectors, and

$$
\begin{aligned}
D_{1}+N_{16}-2 N_{13} \sim & T_{36}-T_{14}+T_{23}+2 N_{26}+3 T_{25}+2 N_{56} \\
+ & T_{15}+N_{24}+T_{46}+N_{45} \\
D_{3}+N_{36}-2 N_{13} \sim & T_{16}-T_{34}+T_{23}+2 N_{26}+3 T_{25} \\
& +2 N_{56}+T_{15}+N_{24}+T_{46}+N_{45} .
\end{aligned}
$$

We see that $D_{1}$ and $D_{3}$ are sections and $D_{1} \oplus N_{16}=D_{3} \oplus N_{36}=0$, where $\oplus$ is the group operation on the set of sections with zero section defined by $N_{13}$. These determine the action of $f_{r}$ as follows:

$$
\begin{aligned}
& N_{16} \longleftrightarrow D_{1}, \quad N_{26} \longleftrightarrow N_{56}, \quad N_{36} \longleftrightarrow D_{3}, \quad N_{46} \longleftrightarrow N_{46}, \\
& N_{12} \longleftrightarrow C_{56}, \quad N_{13} \longleftrightarrow N_{13}, \quad N_{24} \longleftrightarrow N_{45}, \quad N_{35} \longleftrightarrow C_{26}, \\
& T_{15} \longleftrightarrow T_{23}, \\
& f_{r}\left(T_{\beta}\right)=T_{\beta}, \quad \beta=16,36,46,14,25,34, \\
& f_{r}\left(T_{26}\right)=4 \eta_{H}-2\left(T_{26}+T_{56}\right)-T_{26}-T_{15}-T_{23} \\
& -2\left(N_{16}+N_{26}+N_{36}+N_{56}+N_{12}+N_{24}+N_{35}+N_{45}\right) \text {, } \\
& f_{r}\left(T_{56}\right)=4 \eta_{H}-2\left(T_{26}+T_{56}\right)-T_{56}-T_{15}-T_{23} \\
& -2\left(N_{16}+N_{26}+N_{36}+N_{56}+N_{12}+N_{24}+N_{35}+N_{45}\right) \text {. }
\end{aligned}
$$

Note that

$$
D_{1}+D_{3}=N_{16}+N_{36}+6 r_{1},
$$

so that $f_{r}\left(6 r_{1}\right)=-6 r_{1}$. Again $f_{r}$ is not a reflection, but works like a reflection.

The involution $f_{r}$ is not symmetric in the sense that

$$
f_{r}\left(T_{26}+T_{56}\right) \neq T_{26}+T_{56}+6 r_{1} .
$$

On the other hand, the map

$$
g_{r}=g_{N_{16}, N_{36}, T_{26}, T_{56}}:=f_{N_{16}, N_{36}, T_{26}, T_{56}} \circ p_{35} \circ p_{12}
$$

is symmetric. We have

$$
\left[\begin{array}{cccccccccc}
N_{16} & N_{26} & N_{36} & N_{46} & N_{56} & N_{12} & N_{13} & N_{24} & N_{35} & N_{45} \\
D_{3} & N_{45} & D_{1} & N_{13} & N_{24} & N_{12} & N_{46} & N_{56} & N_{35} & N_{26}
\end{array}\right]
$$




$$
\left[\begin{array}{cccccccccc}
T_{16} & T_{26} & T_{36} & T_{46} & T_{56} & T_{14} & T_{15} & T_{23} & T_{25} & T_{34} \\
T_{34} & G_{2} & T_{14} & T_{25} & G_{5} & T_{36} & T_{15} & T_{23} & T_{46} & T_{16}
\end{array}\right]
$$

where $g_{r}$ interchanges two elements in the same column, and

$$
\begin{aligned}
G_{2}= & T_{26}+T_{15}+T_{23}+2\left(T_{46}+T_{25}\right)+2\left(N_{26}+N_{24}\right) \\
& -\left(N_{16}+N_{36}\right)+N_{56}+N_{45}+N_{13}+N_{46}, \\
G_{5}= & T_{56}+T_{15}+T_{23}+2\left(T_{46}+T_{25}\right)+2\left(N_{56}+N_{45}\right) \\
& -\left(N_{16}+N_{36}\right)+N_{26}+N_{24}+N_{13}+N_{46} .
\end{aligned}
$$

This is an isometry of $S_{H}$ acting as -1 on the discriminant form of $S_{H}$, and hence sending $6 r_{1}$ to $-6 r_{1}$. In fact, following an idea from [Ke2], we found first the lattice involution $g_{r}$ and then $f_{r}$ which realizes it geometrically.

Since $g_{r}\left(N_{16}+N_{36}\right)=N_{16}+N_{36}+6 r_{1}$ and $g_{r}\left(T_{26}+T_{56}\right)=T_{26}+T_{56}+6 r_{1}$, we have

$$
g_{r}\left(r_{1}\right)=-r_{1}, \quad g_{r}\left(\omega^{\prime}\right)=\omega^{\prime}+12 r_{1}=\omega^{\prime}+3\left(\omega^{\prime}, r_{1}\right) r_{1},
$$

where $\omega^{\prime}=\sum_{\alpha} N_{\alpha}+\sum_{\beta} T_{\beta}$ is the projection of the Weyl vector.

Automorphisms of type (3b). This is the involution $\tau \circ g_{\tau(r)} \circ \tau$.

8.6. Remark. The translation by the 2-torsion $N_{46}$ is the same as $\tau \circ$ (involution defined by the skew lines $\left.T_{26}, T_{56}\right)=p_{35} \circ p_{12}$.

8.7. Remark. If we take $N_{16}$ as the zero section (and $N_{36}$ a 2-torsion), then the inversion map corresponds to a Leech root of type (3b), more precisely, to $r$ with

$$
\begin{aligned}
& \left(r, N_{\alpha}\right)= \begin{cases}1, & \text { if } \alpha=46,13 ; \\
0, & \text { otherwise },\end{cases} \\
& \left(r, T_{\beta}\right)= \begin{cases}1, & \text { if } \beta=26,56 ; \\
0, & \text { otherwise }\end{cases}
\end{aligned}
$$

8.8. Theorem. The automorphism group of $\tilde{H}$ is generated by the Enriques involution $\tau$, the 10 projections $p_{\alpha}$, the 15 inversion automorphisms $f_{r}$ of elliptic pencils of type 2, and the 12 inversion automorphisms $\phi_{r}$ of elliptic pencils of type 3.

Proof. Let $\sigma_{r}$ be the involution corresponding to a Leech root $r$ of type (1a), (1b), (2), (3a), or (3b), i.e. $\sigma_{r}$ is one of the twelve $\phi_{r}$, twelve $\tau \circ \phi_{\tau(r)} \circ \tau$, ten $p_{r}$, fifteen $g_{r}$, or fifteen $\tau \circ g_{\tau(r)} \circ \tau$. Let $N$ be the subgroup of $\operatorname{Aut}(\tilde{H})$ generated by them.

As we explained before (after Lemma 8.2), the result follows from the following lemma.

8.9. Lemma. Let $\gamma$ be an isometry of the Picard lattice $S_{H}$ which preserves $P\left(S_{H}\right)$. Then there exists an element $\delta \in N$ such that $\delta \circ \gamma \in \operatorname{Aut}\left(P\left(S_{H}\right)^{\prime}\right)$.

Proof. This is similar to the proof of Lemma 7.3 in [Ko]. Take an element $\delta \in N$ which realizes $\min \left\{\left(\delta\left(\gamma\left(\omega^{\prime}\right)\right), \omega^{\prime}\right): \delta \in N\right\}$. Then, for any $r$,

$$
\left(\delta \circ \gamma\left(\omega^{\prime}\right), \omega^{\prime}\right) \leq\left(\sigma_{r} \circ \delta \circ \gamma\left(\omega^{\prime}\right), \omega^{\prime}\right)=\left(\delta \circ \gamma\left(\omega^{\prime}\right), \sigma_{r}\left(\omega^{\prime}\right)\right) .
$$

If $\sigma_{r}=\phi_{r}$ (of type (1a)), then, applying (8.4), we get

$$
\left(\delta \circ \gamma\left(\omega^{\prime}\right), \omega^{\prime}\right) \leq\left(\delta \circ \gamma\left(\omega^{\prime}\right), \omega^{\prime}\right)+15\left(\delta \circ \gamma\left(\omega^{\prime}\right), r_{1}\right) .
$$


This means that $\left(\delta \circ \gamma\left(\omega^{\prime}\right), r_{1}\right) \geq 0$. Since $\omega^{\prime}$ is an interior point of $P\left(S_{H}\right)^{\prime}$, the last inequality is strict.

If $\sigma_{r}=\tau \circ \phi_{\tau(r)} \circ \tau$ (of type (1b)), then, since $\tau$ preserves $\omega^{\prime}$, we have

$$
\sigma_{r}\left(\omega^{\prime}\right)=\tau\left(\omega^{\prime}+15 \tau\left(r_{1}\right)=\omega^{\prime}+15 r_{1},\right.
$$

and hence again $\left(\delta \circ \gamma\left(\omega^{\prime}\right), r_{1}\right)>0$.

The remaining cases can also be easily handled in this way by using (8.5) and (8.6). Now $\left(\delta \circ \gamma\left(\omega^{\prime}\right), r_{1}\right)>0$ for all $r$, so $\delta \circ \gamma\left(\omega^{\prime}\right) \in P\left(S_{H}\right)^{\prime}$.

8.10. Remark. As a general Hessian quartic surface degenerates to a general Jacobian Kummer surface, the involutions of type (1) are refined to become projections and correlations of the Jacobian Kummer, the involutions of type (2) become Cremona transformations related to Göpel tetrads; on a Jacobian Kummer surface there are 10 Göpel tetrads having exactly 3 elements in common with the hexad (the projection of the Leech root $r_{0}$ from (7.1) onto the Picard lattice of the Jacobian Kummer surface is $\frac{1}{4}\left(3 \eta_{K}-2\right.$ hexad $)$ and the projection of a Leech root of type (2) is $\frac{1}{2}\left(\eta_{K}-\right.$ Göpel tetrad), and these two vectors are orthogonal to each other), and the involutions of type (3a) also become Cremona transformations related to Göpel tetrads; there are 15 Göpel tetrads having exactly 2 elements in common with the hexad. It looks quite complicated to recover the involution $f_{r}$ or $g_{r}$ from its corresponding Cremona transformation on the Jacobian Kummer surface. Finally, the 192 new automorphisms [Ke2] on the Jacobian Kummer surface do not correspond to any automorphisms of the Hessian - in other words, as a general Hessian degenerates to a Jacobian Kummer surface, generators of type (3b) are replaced by new generators, which are the 192 automorphisms. Finally, note that in our case all the generators send one of the half-spaces defined by the corresponding hyperplane to the opposite half-space. The new automorphisms of the Jacobian Kummer do not act in this way; they send the half-space to a half-space corresponding to the inverse automorphism.

\section{REFERENCES}

[Ba] H. Baker, Principles of Geometry. Vol III, Cambridge University Press, 1922, 2nd ed. 1954. MR 31:2650a

[Bo] R. Borcherds, Automorphism groups of Lorentzian lattices, J. Algebra 111 (1987), 133-153. MR 89b:20018

[Cos] F. Cossec, Reye congruences, Trans. Amer. Math. Soc. 280 (1983), 737-751. MR 85b:14049

[CD] F. Cossec, I. Dolgachev, Enriques surfaces, Birkhaüser, Boston 1989. MR 90h:14052

[Hu1] J. Hutchinson, The Hessian of the cubic surface, Bull. Amer. Math. Soc., 5 (1889), 282-292.

[Hu2] J. Hutchinson, The Hessian of the cubic surface, II, Bull. Amer. Math. Soc., 6 (1889), 328-337.

[Ke1] J.H. Keum, Every algebraic Kummer surface is the K3-cover of an Enriques surface, Nagoya Math. J., 118 (1990), 99-110. MR 91f:14036

[Ke2] J.H. Keum, Automorphisms of Jacobian Kummer surfaces, Compositio Math., 107 (1997), 269-288. MR 98e:14039

[KK] J.H. Keum, S. Kondō The automorphism groups of Kummer surfaces associated with the product of two elliptic curves, Trans. Amer. Math. Soc., 353 (2001), 1469-1487. MR 2001k:14075

[Ko] S. Kondō The automorphism group of a generic Jacobian Kummer surface, J. Algebraic Geometry, 7 (1998), 589-609. MR 99i:14043

[Ni] V. Nikulin, Integral symmetric bilinear forms and some of their geometric applications, Math. USSR Izv. 14 (1980), 103-167. MR 80j:10031

[PS] I. Piatetski-Shapiro, I.R. Shafarevich, A Torelli theorem for algebraic surfaces of type K3, Math. USSR Izv. 5 (1971), 547-587. MR 44:1666 
[Ro] J. Rosenberg, Hessian quartic surfaces which are Kummer surfaces, math. AG/9903037.

[Sa] G. Salmon, A Treatise on the Analytic Geometry of Three Dimensions, vol. 2, W. Metcalfe and Son, Cambridge, 5th ed., Longmans and Green, London, 1915; reprint, Chelsea, New York, 1965. MR 34:22

[To] J.A. Todd, A representation of the Mathieu group $M_{24}$ as a collineation group, Ann. Mat. Pura Appl. (4) 71 (1966), 199-238. MR 34:2713

[vGe] B. van Geemen, private notes 1999.

Department of Mathematics, University of Michigan, Ann Arbor, Michigan 48109

E-mail address: idolga@umich.edu

Korea Institute for Advanced Study, 207-43 Cheongryangri-dong, Dongdaemun-gu, Seoul 130-012, Korea

E-mail address: jhkeum@kias.re.kr 